\title{
Fasudil ameliorates cognitive deficits, oxidative stress and neuronal apoptosis via inhibiting ROCK/MAPK and activating Nrf2 signalling pathways in APP/PS1 mice
}

\author{
Wenyue Wei ${ }^{1,2}{ }^{3 *}$, Yuyin Wang ${ }^{2,3 *}$, Jing Zhang ${ }^{2,3}$, Qingfang Gu3 , Xiaoqin Liu ${ }^{3}$, Lijuan Song ${ }^{1,2}$, Zhi Chai ${ }^{1,2,3}$, \\ Minfang Guo ${ }^{3}$, Jiezhong $\mathrm{Yu}^{1,3,4}$, Cungen $\mathrm{Ma}^{1,2,3}$ \\ ${ }^{1}$ Department of Neurology, First Affiliated Hospital, Shanxi Medical University, Taiyuan, P.R. China, ${ }^{2}$ Research Center of \\ Neurobiology, The Key Research Laboratory of Benefiting Qi for Acting Blood Circulation Method to Treat Multiple Sclerosis of State \\ Administration of Traditional Chinese Medicine, Shanxi University of Chinese Medicine, Jinzhong 030619, P.R. China, ${ }^{3}$ Institute of \\ Brain Science, Shanxi Key Laboratory of Inflammatory Neurodegenerative Diseases, Shanxi Datong University, Datong, P.R. China, \\ ${ }^{4}$ Department of Neurology, Datong Fourth People's Hospital, Datong, P.R. China
}

*Wenyue Wei and Yuyin Wang contributed equally to this work.

\begin{abstract}
Alzheimer's disease (AD) is a severe neurodegenerative disorder of the central nervous system (CNS) characterized by neuron loss and dementia. Previous abundant evidence demonstrates that the first critical step in the course of $A D$ is the state of oxidative stress and the neuronal loss is closely related to the interaction of several signalling pathways. The neuroprotective efficacy of Rho-associated protein kinase (ROCK) inhibitor in the treatment of AD has been reported, but its exact mechanism has not been well elucidated. The purpose of this study is to investigate the therapeutic effects of Fasudil on amyloid precursor protein/presenilin-1 (APP/PS1) mice and to discover the potential underlying mechanism. Sixteen 8-month-old APP/PS1 mice were divided into model and Fasudil treatment groups and 8 wild-type mice were used as a normal control group. After the behavioural test, all mice were sacrificed for immunofluorescence and other biochemical tests. The results showed that the administration of Fasudil improved learning and memory ability, elevated the concentration of antioxidative substances and decreased lipid peroxides, as well as inhibited neuronal apoptosis by increasing the expression of B-cell lymphoma-2 $(B C-2)(p<0.05)$, reducing Bcl-2Associated X (Bax) $(p<0.05)$ and cleaved caspase-3 $(p<0.05)$ of APP/PS1 mice. Moreover, Fasudil treatment also ameliorated the phosphorylation of $p 38(p<0.01)$, $c$-Jun N-terminal kinase (JNK) $(p<0.001)$ and extracellular regulated protein kinases $(E R K)(p<0.001)$, and accelerated the nuclear factor-erythroid $2 p 45$-related factor $2(\mathrm{Nrf} 2)(p<0.01)$ expression and its antioxidative downstream molecules $(p<0.05, p<0.05$, and $p<0.05$, respectively). Data from the present study demonstrate that Fasudil significantly restored cognitive function, restrained oxidative stress and reduced neuronal apoptosis in the hippocampus, probably by inhibiting ROCK/MAPK and activating Nrf2 signalling pathways in APP/PS1 mice.
\end{abstract}

Key words: Alzheimer's disease, oxidative stress, apoptosis, ROCK, MAPK, Nrf2.

Communicating authors:

Prof. Cungen Ma, Research Center of Neurobiology, The Key Research Laboratory of Benefiting Qi for Acting Blood Circulation Method to Treat Multiple Sclerosis of State Administration of Traditional Chinese Medicine, Shanxi University of Chinese Medicine, Jinzhong 030619, P.R. China, e-mail: macungen@sxtcm.edu.cn; Prof. Jiezhong Yu, Datong Fourth People’s Hospital, 16 Gongnong Road, Datong, 037009, P.R. China, e-mail: sxdtyjz@qq.com; Prof. Minfang Guo, Institute of Brain Science, Shanxi Key Laboratory of Inflammatory Neurodegenerative Diseases, Shanxi Datong University, 1 Xingyun Road, Datong, 037009, P.R. China, e-mail: guominfang103@163.com 


\section{Introduction}

Alzheimer's disease (AD) is a common neurodegenerative disease, characterized by senile plaques (SP), neurofibrillary tangles (NFTs) and memory deficits as well as behavioural disability [7]. According to the World Alzheimer Report 2016, an estimated 46.8 million individuals suffered from AD-related dementia worldwide and this figure may be forecasted to reach 131.5 million by 2050 [26]. With the coming of world population aging, $A D$, one disease closely related to aging, has become a serious health and economic burden in the society.

Several decades of relevant observations have proved that various pathological changes have occurred during the progression of $A D$ including oxidative stress, mitochondria dysfunction, and neuronal apoptosis, which are likely to have the prominent roles contributing the pathogenesis of AD [17]. Even today, some symptomatic treatments have been taken the clinically, but there is still no effective etiological therapy available [35]. Oxidative stress, as a characteristic of the aging progress, occurs when the level of oxidation exceeds endogenous antioxidant defence, and causes irreversible alternations to biomacromolecule. It is well known that oxidative stress plays a crucial role in pathogenesis of $A D$ on account of its close correlation with the disease severity [27]. Additionally, brain is the most vulnerable region because of its high oxygen consumption and the relatively weak antioxidant system [9]. Particularly, hippocampus, a vital memory centre, would probably be the first brain area with cell death occurring in $A D$ affected by the oxidative damage [25]. These studies provide a novel approach to attenuate neuronal apoptosis and slow down the course of $A D$ by inhibiting oxidative stress response [18]. The neural apoptosis in $A D$ is regulated by various signalling pathways. Primarily, the important serine-threonine Rho-associated protein kinase (ROCK) participates in the regulation of various cellular activities, including proliferation, adherence, contraction, secretion, and apoptosis. ROCK contains two isoforms, ROCK1 and ROCK2, and they share quite high homology. ROCK1 is expressed mainly in the non-neuronal tissue, while the expression of ROCK2 is mainly found in the nervous system [24]. There is considerable evidence indicating that ROCK2 overexpression contributes to the development and progression of neurodegenerative diseases, such as multiple sclerosis (MS), Parkinson's disease (PD), and AD [6]. Thus, inhibiting ROCK2 would be a novel ther- apeutic approach against neurological disorders [28]. Next, the mitogen-activated protein kinase (MAPK) is another kind of serine-threonine kinase that generally exists in eukaryotic cells, and accumulating studies have shown that MAPK pathway activation is closely related with the physiological and pathological process of cells [1]. MAPK is mainly composed of p38 MAPK, c-Jun N-terminal kinase (JNK), and extracellular regulated protein kinases (ERK) [21]. Furthermore, insufficient activation of nuclear factor-erythroid 2 p45-related factor 2 (Nrf2) in the nuclei has also been closely related to chronic neurological disorders such as AD [11]. Nrf2 is the most important redox-regulated transcription factor that plays a pivotal role in anti-oxidative stress via modulating the expression of a battery of endogenous redox-regulated enzymes such as hemeoxygenase-1 $(\mathrm{HO}-1), \mathrm{NAD}(\mathrm{P}) \mathrm{H}$ : quinone oxidoreductase 1 (NQO1), and superoxide dismutase (SOD) [2].

Fasudil, a selective ROCK inhibitor, has been applied to relieve cerebral vasospasm in clinical practice since the last century [22]. Our previous results and other researchers' studies also provided several lines of evidence that ROCK inhibitors significantly improved cognition in amyloid precursor protein/presenilin-1 (APP/PS1) mice, combined with the reduction of pathological products in the whole brain, such as amyloid- $\beta$ (A $\beta$ ) deposits, $p$-Tau and $\beta$-site APP-cleaving enzyme (BACE). In addition, the administration of ROCK inhibitors boosted the synapse function and neurotrophic factors levels, as well as restrained the immune response in the central nervous system (CNS) by regulating the peripheral immune system $[15,30,39]$. And based on the above, Fasudil may be a promising drug for CNS disorders including AD. However, it is still unclear whether Fasudil has antioxidant effects on AD as well as its exact mechanisms. Few studies have focused on the molecular mechanism of Fasudil on $A D$ in vivo involving modulation of MAPK and Nrf2 pathways. Therefore, in this study, we have aimed to investigate the antioxidant effects of Fasudil on the classic mouse model of AD, and explore its possible mechanisms.

\section{Material and methods \\ Animals}

Male APP/PS1 double transgenic mice, three-monthold, and age- and sex-matched C57BL/6 mice were purchased from Beijing HFK Bioscience Co., Ltd. All mice were raised under specific pathogen-free conditions 
of lighting (12 h/12 h light/dark cycle), temperature $\left(25 \pm 2^{\circ} \mathrm{C}\right)$, and relative humidity $(50 \pm 10 \%)$. The mice were provided with unlimited diet and water. All experimental procedures were conducted according to the guidelines of the International Council for Laboratory Animal Science and were approved by the Ethics Committee of the Shanxi Datong University, Datong, China The APP/PS1 double transgenic mice were randomly divided into two groups: Fasudil group (F group, $n=8$ ) and normal saline group (NS group, $n=8$ ). And the wild type mice served as a control group (WT group, $n=8$ ).

\section{Drug treatment}

Fasudil (30 mg: $2 \mathrm{ml}$ per ampoule) were obtained from Tianjin Chasesun Pharmaceutical Co. and dissolved in a sterile saline solution. Starting at the age of eight months, the $\mathrm{F}$ group mice were intraperitoneally injected with Fasudil (25 mg/kg per day) for 2 months, while the NS group and WT group were treated with equivalent normal sodium $(0.9 \% \mathrm{NaCl})$. The dosage of Fasudil was selected based on our previous reports [38].

\section{Morris Water Maze test}

To evaluate spatial learning and memory abilities of mice, we performed the following procedure as described earlier [39]. The Morris Water Maze (MWM) is a round pool ( $90 \mathrm{~cm}$ in diameter), containing a platform ( $5 \mathrm{~cm}$ in diameter) in the centre of $1 / 4$ quadrant of the maze below the water surface. The tank was filled with water containing titanium dioxide maintained at around $19^{\circ} \mathrm{C}$. Mice underwent training for 5 consecutive days of twice a day in the pool before the formal test. If the mouse failed to arrive at the target within 60 seconds, it would be guided to the target and permitted to stop briefly around $10 \mathrm{sec}$ onds. The movements of mice were monitored by the automated analysis system (SMART V3.0 system, Panlab, Barcelona, Spain). The escape latency, the distance percentage in the SW target quadrant and swim speed were analysed during the test.

\section{Y-maze test}

The Y-maze test was based on the method described by Burrows et al. with minor modifications [4]. The apparatus, used to measure short-term memory, consisted of three symmetrical opaque arms $(30 \mathrm{~cm}$ long, $8 \mathrm{~cm}$ wide, and $15 \mathrm{~cm}$ high) at a quiet room under dim light. Applying the principle of randomness, we named three arms as the start arm, the novel arm, and the other arm. Different visual cues were used on the surrounding walls. Within $10 \mathrm{~min}$, the mouse was placed in the maze to explore the start and the other arm accompanied by the novel arm blocked. After a one-hour delay, the formal trial was conducted and the mouse was again put into the same starting arm, with all three arms open for $5 \mathrm{~min}$. The sawdust was replaced and the maze was cleaned with $70 \%$ ethanol to minimize the interference of smell between each trial. By using the automated SMART V3.0 system, all trials were recorded, and the percentage of time spent in the novel arm and alternation were measured in order to detect the exploration ability and spatial recognition memory.

\section{Perfusion and tissue preparation}

After finishing all behaviour tests, half of the mice in each group $(n=4)$ were anaesthetized and perfused intracardially with cold normal saline. The brains used for experiments were immediately isolated. And the hippocampus tissue was dissected out by using a dissecting microscope and homogenized in RIPA buffer (KeyGEN) with protease inhibitors (KeyGEN) and centrifuged at $12000 \mathrm{rpm}$ for $10 \mathrm{~min}$. The BCA kit (Solarbio) was used to determine the concentration of the hippocampus extract. The remaining mice $(n=4)$ were perused with saline and $4 \%$ paraformaldehyde (PFA) in phosphate buffer (PBS, $0.01 \mathrm{M}$, $\mathrm{pH}=7.4)$. The brains were collected and frozen in liquid nitrogen. Consecutive coronal sections were sliced using a cryostat microtome (Leica) at $10 \mu \mathrm{m}$.

\section{Measurement of substances associated with oxidative stress}

Commercial assay kits (SOD: S0101, Beyotime Biotechnology; GSH: A061-1, NanJing JianCheng Bioengineering; MDA: S0131, Beyotime Biotechnology) were used to measure the concentrations of superoxide dismutase (SOD), glutathione (GSH), and malondialdehyde (MDA) in hippocampal tissue according to manufacturers' instructions, respectively.

\section{Western Blot analysis}

Total $20 \mu \mathrm{g}$ of samples were separated by sodium dodecyl sulfate-polyacrylamide gel electrophoresis (SDS-PAGE) and transferred onto polyvinylidene fluoride (PVDF) membrane (Millipore), and then 
blocked at room temperature (RT) with $5 \%$ non-fat milk for 2 hours and incubated at $4^{\circ} \mathrm{C}$ overnight with the following primary antibodies: Anti-ROCK2 (Abcam), Anti-ROCK2 (phospho S1366, Abcam), Anti-Bax (Abcam), Anti-Bcl-2 (Abcam), Anti-Cleaved Caspase3 (Abcam), Anti-p38 (phospho Y182, Abcam), Anti-JNK1+JNK2+JNK3 (phospho T183+T183+T221, Abcam), Anti-ERK (phospho Tyr204, Santa Cruze), Anti-Nrf2 (Abcam), Anti-HO-1 (Abcam), Anti-NQO1 (Abcam) and Anti-SOD2 (Cell Signaling). After three rinses in TBST, the immunoblots were incubated at RT for 2 hours with HRP-conjugated secondary antibodies (Cell Signaling), washed again and visualized by ECL (Millipore). Anti-GAPDH (Cell Signaling) was taken as a loading control. And Image Lab Software (Bio-rad Laboratories) was applied to quantify the intensity of the protein bands. The experiment was repeated three times.

\section{Immunohistochemistry analysis}

Brain slides were blocked and permeabilized with $0.3 \%$ Triton $\mathrm{X}-100$ in $1 \%$ BSA/PBS for 1 hour at RT, then incubated at $4^{\circ} \mathrm{C}$ overnight with primary antibodies as follows: Anti-NeuN (Abcam), Antip-ROCK2 (phospho S1366, Abcam), Anti-Bax (Abcam), Anti-Bcl-2 (Abcam), Anti-Cleaved Caspase3 (Abcam), Anti-p38 (phospho Y182, Abcam), AntiJNK1+JNK2+JNK3 (phospho T183+T183+T221, Abcam), Anti-ERK (phospho Tyr204, Santa Cruze), Anti-Nrf2 (Abcam), Anti-HO-1 (Abcam), Anti-NQO1 (Abcam) and Anti-SOD2 (Cell Signaling). After washing with PBS, corresponding secondary antibodies ( 1 : 1000, Cell Signaling) were applied at RT for $2 \mathrm{~h}$. Then, the slides were coverslipped with a mounting medium containing 4',6-diamidino-2-phenylindole (DAPI). The negative control sections were treated using the same protocols, but omitting the primary antibodies. Three consecutive sections per mouse were observed under a confocal microscope (Olympus FV1000) in a blinded fashion. And the area (polygon) of double positive cells were quantitatively analysed by Image-Pro Plus software. All sections were double-blindly examined.

\section{TUNEL assay}

Terminal deoxynucleotidyl transferase dUTP nick end labelling (TUNEL) is the sensitive and reliable way to examine apoptosis. A One Step TUNEL Apoptosis Kit (Beyotime) was used to observe neuronal in situ apop- tosis in the hippocampus region according to the manufacturer's recommendations. Image-Pro Plus software was used to count the number of TUNEL ${ }^{+}$cells.

\section{ROCK2 activity assay}

The ROCK2 activity in the supernatant of mice hippocampus homogenates was determined by a Rho kinase testing kit (GMS50184.2) according to the manufacturer's instructions.

\section{Statistical analysis}

All statistics were analysed by GraphPad Prism 5.0 software (GraphPad software, San Diego, CA), and all of the data were expressed as mean \pm standard deviation (SD). Behavioural data for escape latency were compared by two-way analysis of variance (ANOVA). Differences between the groups were compared by one-way ANOVA followed by Tukey's post-hoc test. The statistical method was also described in the Figure legend. The $p$ value of less than 0.05 was defined statistically significant.

\section{Results}

\section{Fasudil ameliorated cognitive impairment in APP/PS1 mice}

The MWM and Y-maze tests were performed to assess the beneficial effects of Fasudil on the cognitive function in APP/PS1 mice after two-month treatment. Figure $1 \mathrm{~A}$ has shown the schematic diagram of 8-zone MWM test and representative path tracking of each group. There was no significant difference in the time taken by mice to locate the hidden platform between groups during the five-day initial training session of the MWM test $(p>0.05)$ as shown in Figure 1B. However, we have observed significant differences in the subsequent formal tests. Compared with the control WT mice, escape latency in the NS group was significantly prolonged ( $p<0.01$, Fig. 1C) and the distance percentage in the SW zone exhibited partial reduction $(p<0.01$, Fig. 1D), representing that the APP/PS1 transgenic mice had a typical cognitive dysfunction. However, this dysfunction was partly reversed by Fasudil treatment, with shorter escape latency and longer distance in the SW zone when compared with the NS group ( $p<0.05$, and $p<0.05$, respectively), and there was no significant change in the Fasudil group when compared with those of the WT group $(p>0.05$, 
A

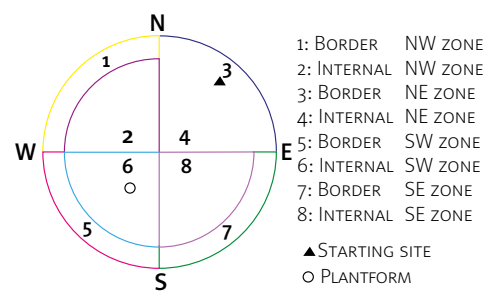

NS
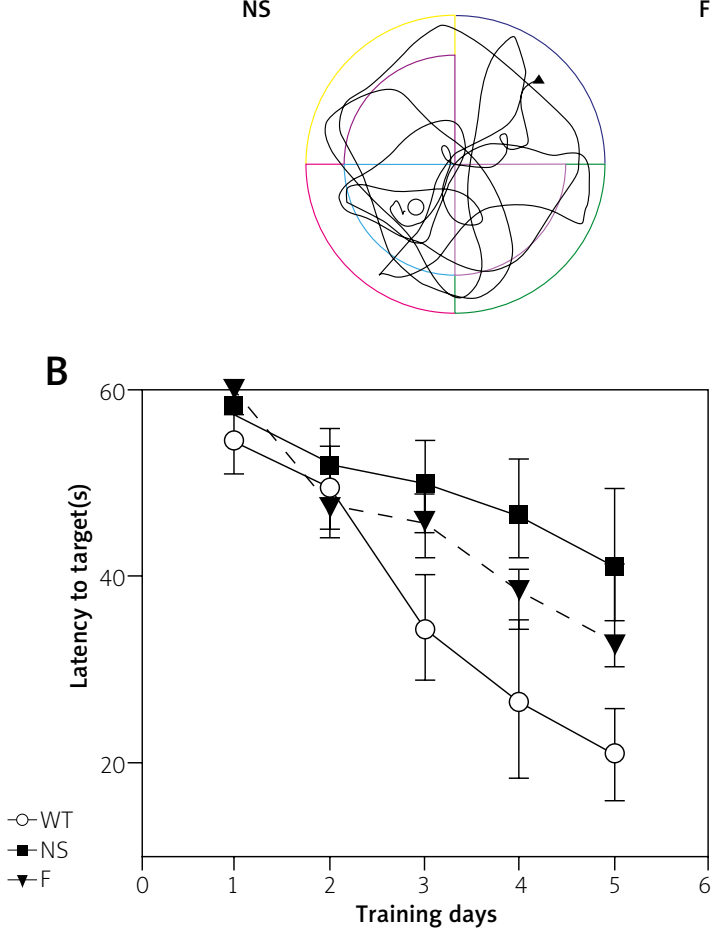

D

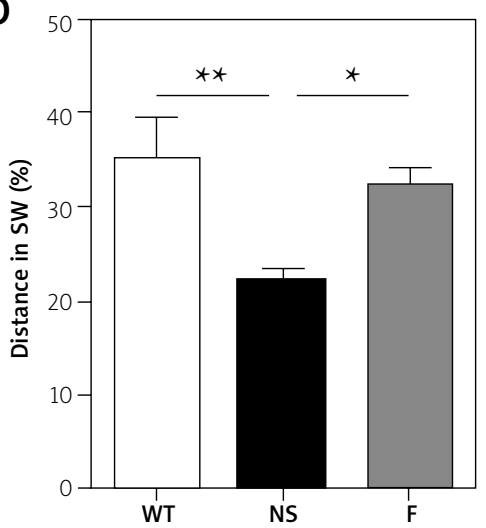

WT

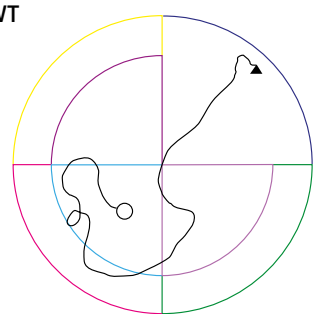

F
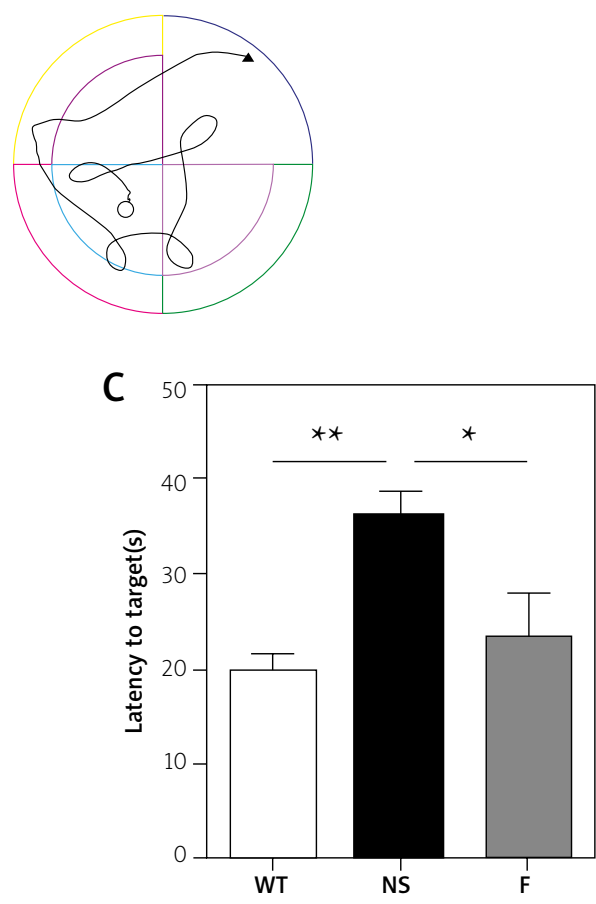

E

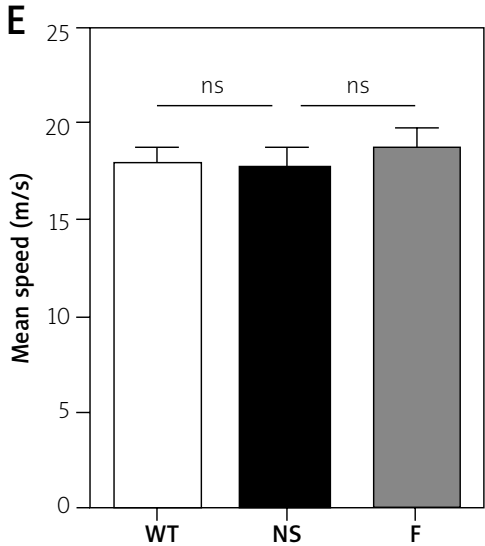

Fig. 1. Fasudil improved learning and memory ability of APP/PS1 mice. Eight-month-old APP/PS1 mice received Fasudil $(n=8)$ or saline $(n=8)$ by intraperitoneal injection for 2 months. C57BL/6 mice of the same age and gender $(n=8)$ were taken as control mice, injected with normal saline (NS). The Morris water maze (MWM) and Y-maze tests were used to evaluate learning and memory ability, and assess the exploratory activities of mice. A) The schematic diagram of 8-zone MWM test and representative path tracking of each group. B) The time spent by mice from the starting point to the submerged platform on the five consecutive days for daily tests (two-way ANOVA). C) The time spent searching for the target during the formal test. D) The percentage of distance in the SW zone during the formal test. E) The swim speed during the formal test. 

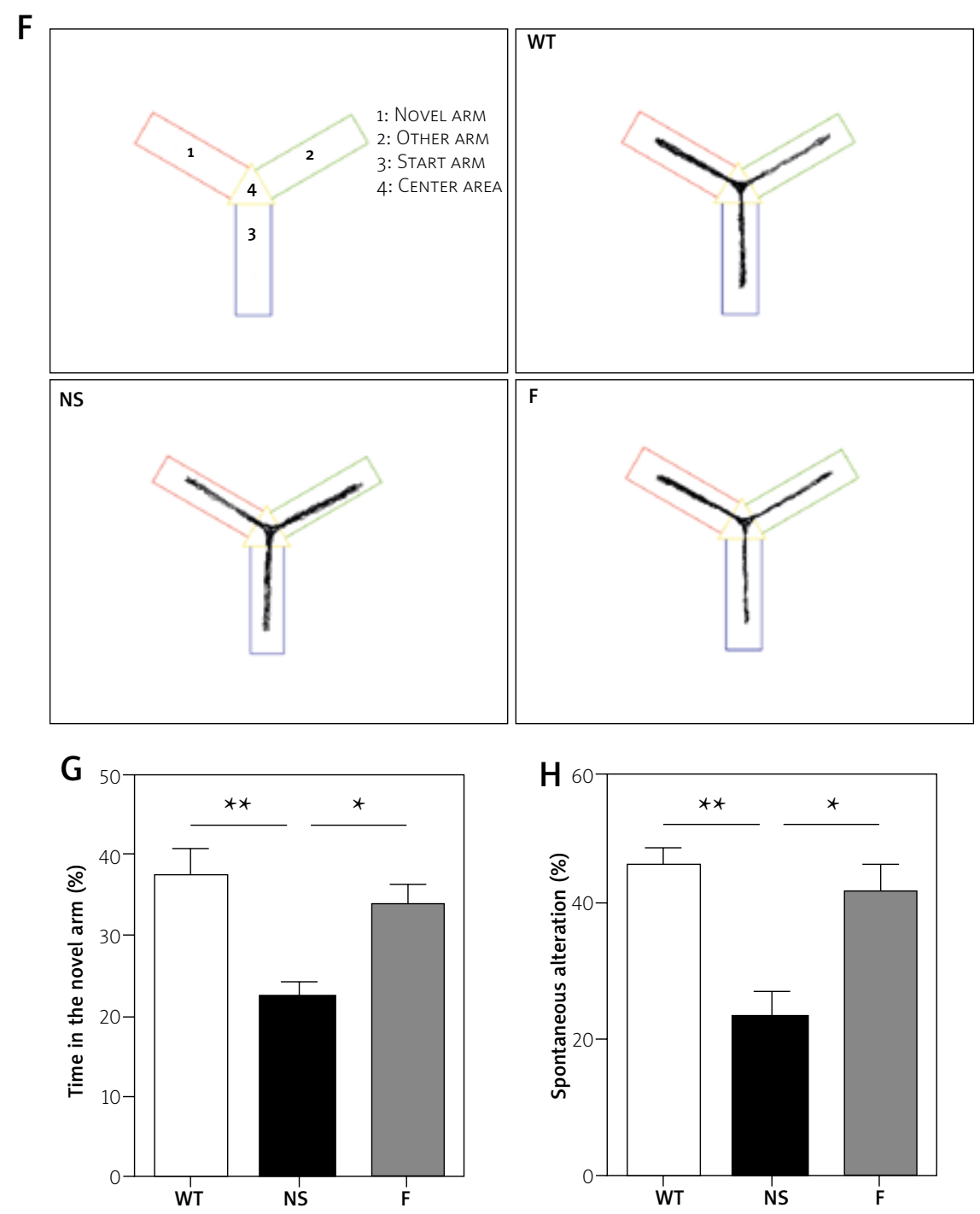

Fig. 1. Cont. F) The schematic diagram of Y-maze and tracing of movement of each group. G) The percentage of time spent in the novel arm assessing the exploratory activities of mice. $\mathrm{H}$ ) The definition of alternation is the consecutive entries into all three arms. Spontaneous alternation is the ratio of the number of alternations to the total possible alternations, which reflects spatial working memory. Results were shown as mean \pm SD of 8 mice per group. Significant differences between groups were presented as ${ }^{*} p<0.05$, ${ }^{* *} p<0.01,{ }^{* *} p<0.001$, one-way ANOVA followed by Tukey's post-hoc test.

and $p>0.05$, respectively). Additionally, there were no differences in mean swim speed among three groups ( $p>0.05$, Fig. $1 \mathrm{E}$ ), revealing that observed differences were not caused by the physical handicap of mice. Besides the MWM trial, we further conducted Y-maze test to assess the level of exploratory activity and the spatial working memory of the mice. The time spent in the novel arm was significantly shorter ( $p<0.01$, Fig. 1G) and the percentage of the spontaneous alteration was lower $(p<0.01$,
Fig. $1 \mathrm{H}$ ) in NS vehicle-treated APP/PS1 mice than in the wild type mice, and the Fasudil treatment increased the time in the novel arm $(p<0.05)$ and the spontaneous alteration $(p<0.05)$ in APP/PS1 mice and even similar to the levels in the WT group $(p>0.05)$. Hippocampus is well known as the memory region which is closely associated with cognition in the brain. Therefore, these data showed that the Fasudil administration could rescue the function of hippocampus and has a great influence on both learning and memory in APP/PS1 Tg mice. 


\section{Fasudil attenuated oxidative stress in APP/PS1 mice}

There has been a huge amount of researches demonstrating that the effects of oxidative stress plays a vital role in the course of $A D$, involving excessive oxidative damage and impaired antioxidant ability [31]. In order to confirm the antioxidant properties of Fasudil in APP/PS1 mice, we measured the levels of oxidative stress-related characteristic biomarkers, such as SOD, total glutathione (T-GSH), oxidized glutathione (GSSG), GSH, and MDA. As shown in Figure $2 \mathrm{~A}$, the concentrations of SOD $(p<0.001)$, T-GSH $(p<0.001)$, GSSG $(p<0.001)$, and GSH $(p<0.001)$ were obviously decreased in the APP/PS1 group as compared with those of the WT group. But these changes were all significantly reversed by Fasudil treatment ( $p<0.01, p<0.01, p<0.01$, and $p<0.01$, respectively). Although the levels of SOD were declined $(p<0.01)$ in the Fasudil treatment group, there were no significant differences in the levels of T-GSH, GSSG, and GSH, when compared to the WT group $(p>0.05)$. Moreover, a higher level of MDA ( $p<0.05$, Fig. 2B) was observed in the APP/PS1 group compared with the WT group, while Fasudil administration dramatically inhibited the elevation of this lipid peroxidation marker $(p<0.001)$ even more than in the WT group $(p<0.05)$. All of the results showed that Fasudil strengthened the antioxidant system and suppressed oxidative stress response in APP/PS1 mice.

\section{Fasudil reduced neuronal apoptosis in APP/PS1 mice}

Our previous results showed that Fasudil had neuroprotective effects [10]. Therefore, we evaluated the effects of Fasudil on neurons by detecting apoptotic markers and apoptosis-related proteins, such as TUNEL positive neuronal cells and the level of Bax, $\mathrm{Bcl}-2$, and cleaved caspase-3 in APP/PS1 mice.
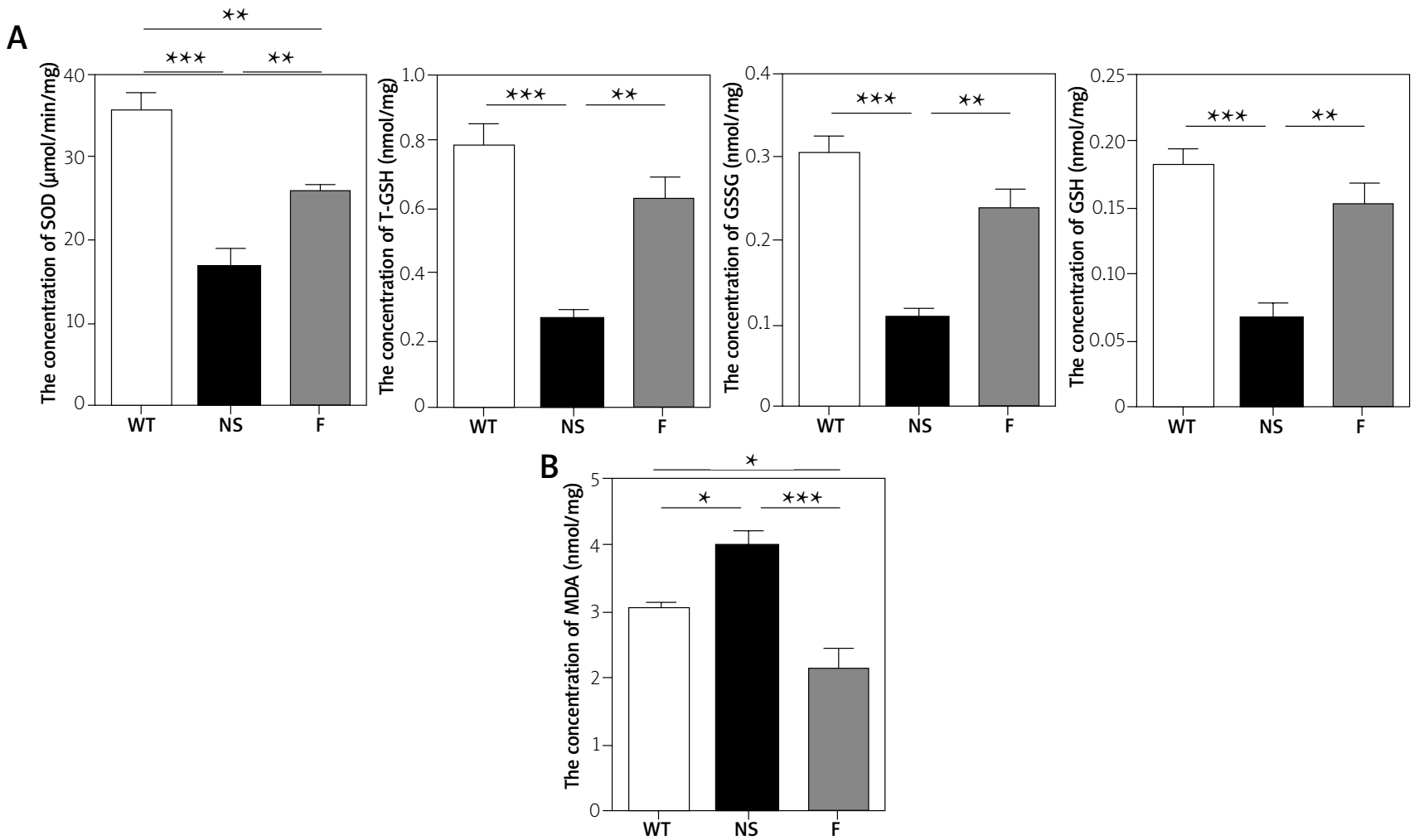

Fig. 2. Fasudil increased the contents of antioxidant enzymes and attenuated the oxidative related factors in the hippocampus of APP/PS1 mice. Mice were sacrificed after finishing all behaviour tests and the brains were isolated for subsequent analysis. A) The levels of antioxidants, including SOD, GSH, GSSG, and T-GSH, in the supernatant of hippocampus homogenates among three groups were measured by commercial kits. B) A representative bar graph showed the content of MDA in the hippocampus lysate, which represented the production of lipid peroxide. The graphs were expressed as the mean \pm SD, $n=4$ per group. Significant differences between groups were presented as ${ }^{*} p<0.05,{ }^{* *} p<0.01,{ }^{* * *} p<0.001$, one-way ANOVA followed by Tukey's post-hoc test. 
As shown in Figure 3A, the number of TUNEL-positive neuronal cells was increased $(p<0.001)$ and pathological changes of neurons occurred in APP/PS1 mice in immunofluorescence staining of hippocampus tissue compared with the normal control group. The neurons in the model group showed irregular arrangement and severe degeneration, including swelling, necrosis, pyknosis and karyorrhexis. In addition to these pathological changes, as shown in Fig. 3B and C, our Western blot analysis and immunofluorescence staining indicated the increases in $\operatorname{Bax}(p<0.001$, and $p<0.001$, respectively), the ratio of $\mathrm{Bax}$ to $\mathrm{Bcl}-2$ $(p<0.001)$, and cleaved caspase- $3(p<0.001$, and $p<0.05$, respectively) and the decrease in Bcl-2 ( $p<0.05$, and $p<0.001$, respectively) in the APP/ PS1 mice compared with WT mice. Fasudil administration increased the expression of $\mathrm{Bcl}-2(p<0.05$, and $p<0.05$, respectively) and reduced the expression of cleaved caspase- $3(p<0.05$, and $p<0.05$, respectively) compared with APP/PS1 mice and the levels were similar to the results of WT mice $(p>0.05)$. And although the number of TUNEL-positive cells $(p<0.01)$, the level of $\operatorname{Bax}(p<0.05$, and $p<0.05$, respectively) and the ratio of $\mathrm{Bax}$ to $\mathrm{BCl}-2$ $(p<0.01)$ were slightly increased compared with those of the WT group, the levels were significantly decreased compared with the NS group $(p<0.001, p<0.01, p<0.001$, and $p<0.001$, respectively). These results suggested that Fasudil exerts powerful anti-apoptotic effects on $A D$ through initiating apoptotic signalling cascades, reducing the expression of pro-apoptotic Bax and cleaved caspase-3, and improving the level of anti-apoptotic Bcl-2.

A
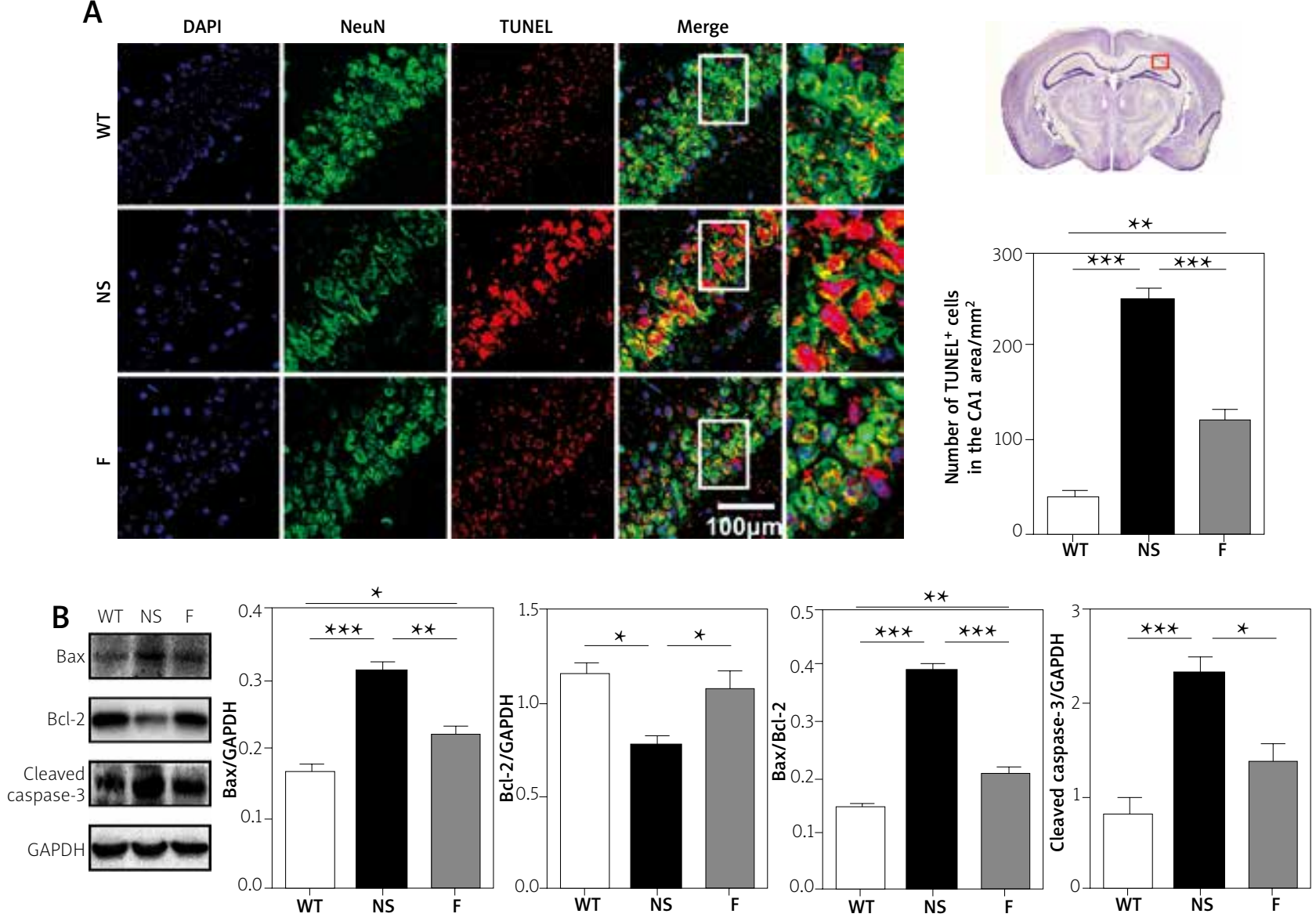

Fig. 3. Fasudil inhibited hippocampus neuron apoptosis. A) Representative confocal images of TUNEL ${ }^{+}$cells in the CA1 region of hippocampus showed the severity of neuronal apoptosis (40x, Scale bar: $100 \mu \mathrm{m}$ ). B) Western blot bands and statistical results showed the changes of the levels of apoptosis-related proteins such as Bax, BCl-2, and cleaved caspase-3. GAPDH was used as an internal loading control. 
C
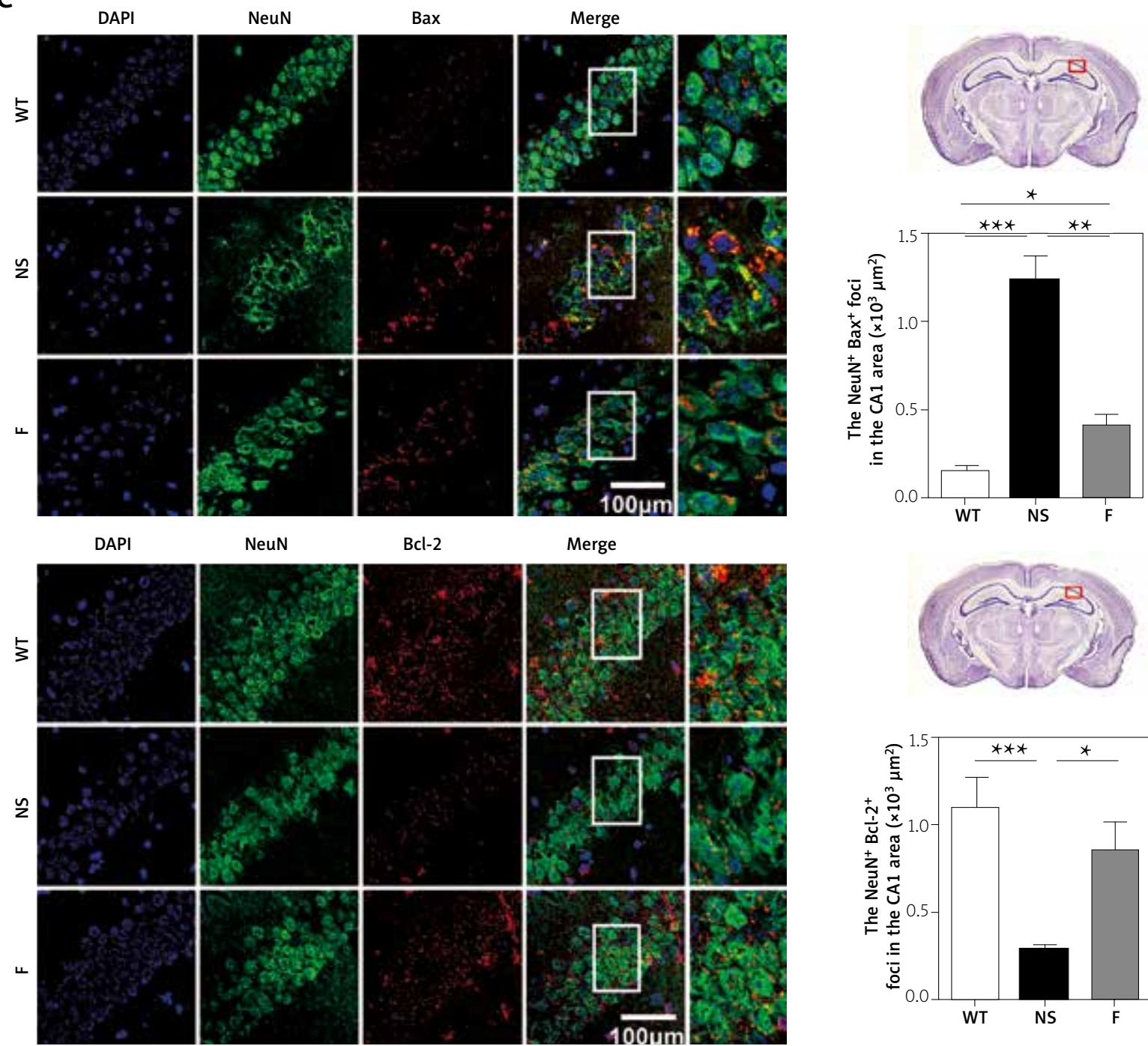

DAPI

NeuN

Caspase-3

Merge
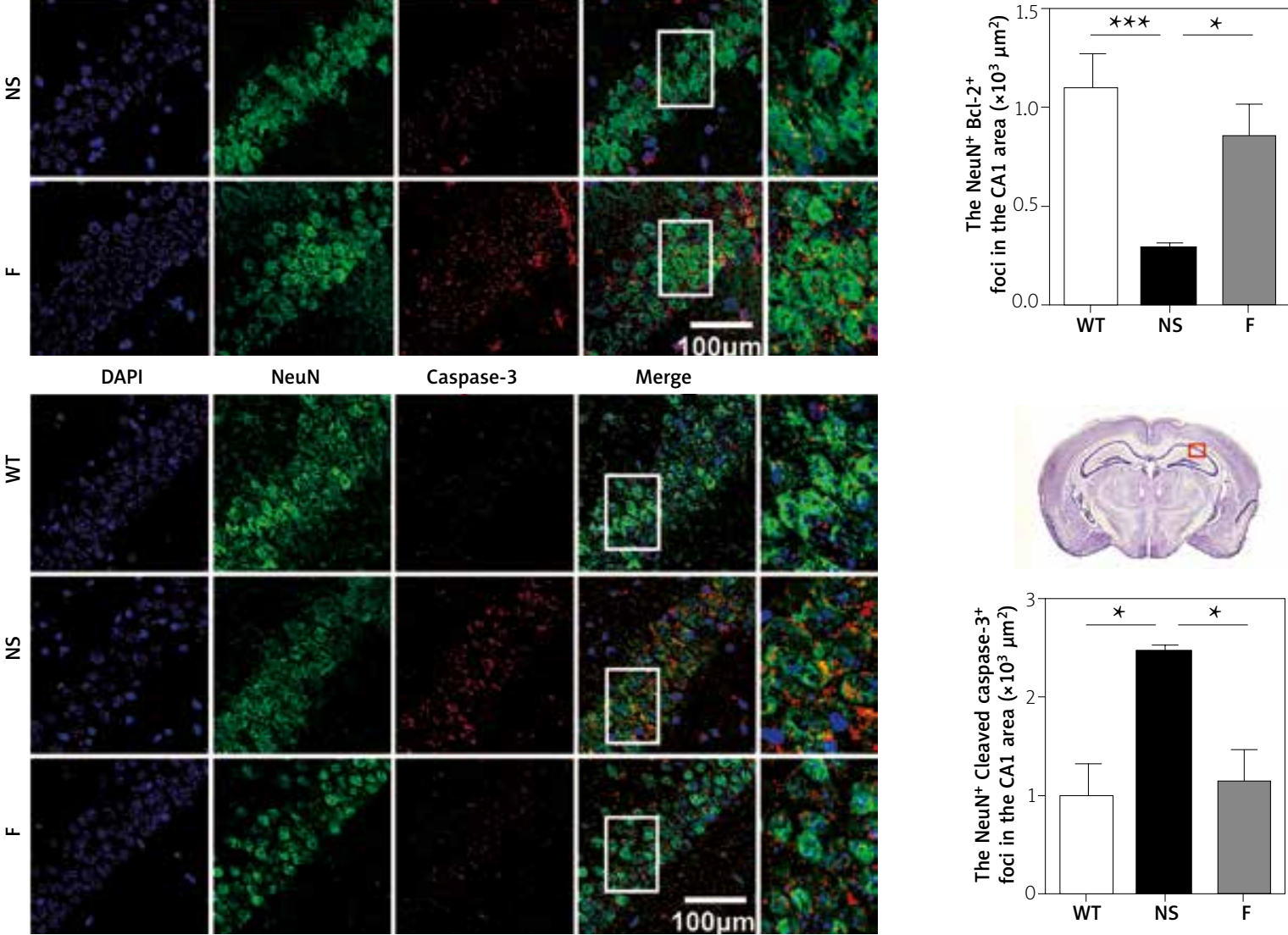

Fig. 3. Cont. C) The representative images of Bax+, Bcl-2+, and cleaved caspase-3+ (red) in neurons (green) of three groups by double immunofluorescence and quantitative analysis (40x, Scale bar: $100 \mu \mathrm{m})$. Data were expressed as mean $\pm S D, n=4$ per group. Significant differences between groups were presented as ${ }^{*} p<0.05$, ${ }^{* \star} p<0.01,{ }^{* \star \star} p<0.001$, one-way ANOVA followed by Tukey's post-hoc test. 

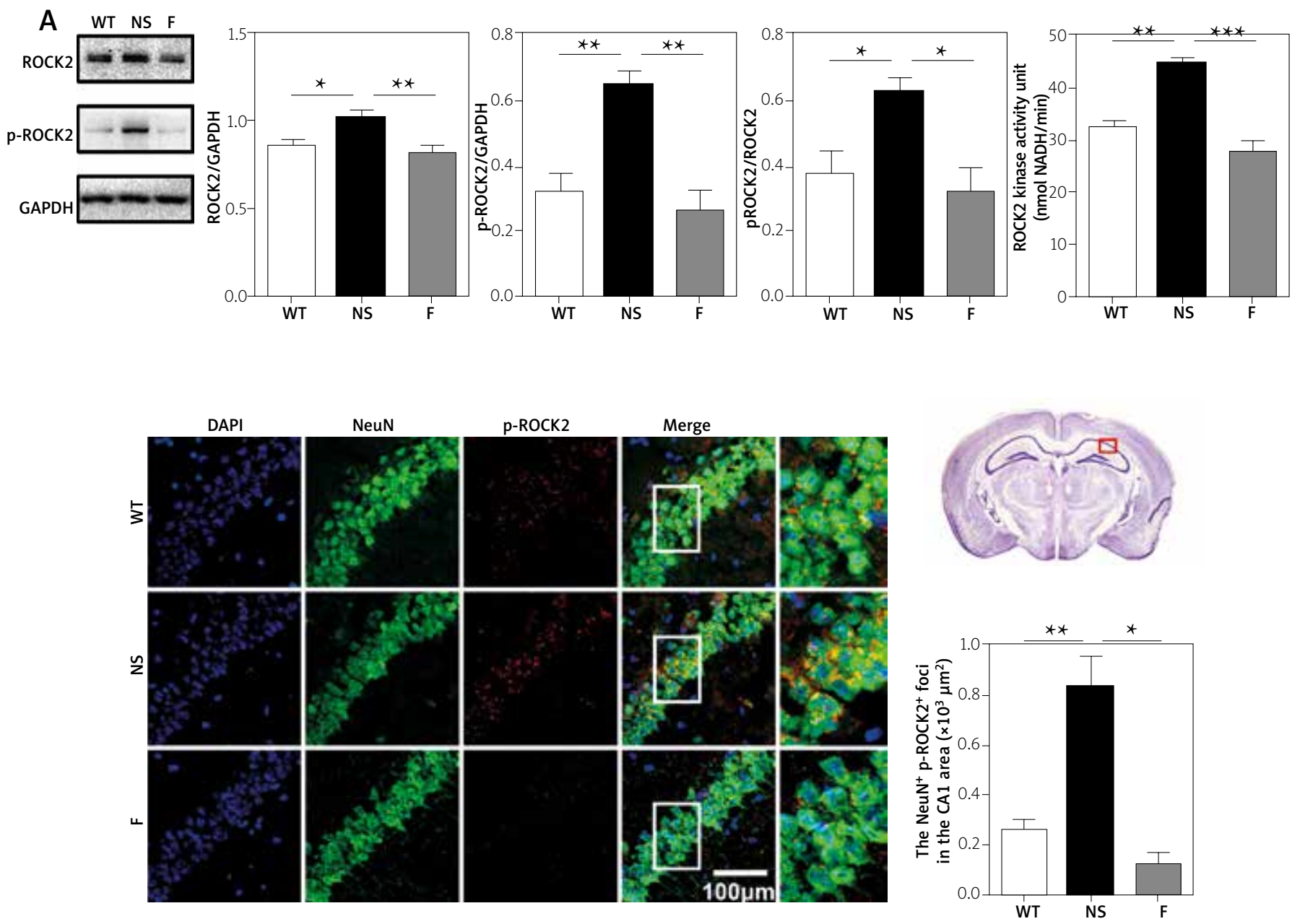

Fig. 4. Fasudil restrained the activity and expression of Rho kinase. A) The levels of ROCK2 and p-ROCK2 (S1366) were detected by Western blot analysis. The levels of quantitative proteins were normalized to the levels of GAPDH. B) The activity of ROCK2 was measured by a kit. C) p-ROCK2 (S1366) (red) positive neurons (green) in CA1 zone of the hippocampus in mice of three groups by double immunofluorescence (40x, Scale bar: $100 \mu \mathrm{m})$. The area (polygon) of NeuN+ p-ROCK (S1366)+ cells in the hippocampus was analysed quantitatively by Image-Pro Plus software. Data were expressed as mean $\pm S D, n=4$ per group. Significant differences between groups were presented as ${ }^{*} p<0.05,{ }^{* *} p<0.01,{ }^{* * *} p<0.001$, one-way ANOVA followed by Tukey's post-hoc test.

\section{Fasudil down-regulated expression and activity of ROCK2 in APP/PS1 mice}

During the past decade, a large number of studies demonstrated that ROCK2, which is mainly expressed in the brain, played a vital role in the pathological process of AD [8]. In the current study, compared with WT mice, we discovered that the level of p-ROCK2 (S1366) was enhanced in APP/PS1 mice, which was identified by both Western blot $(p<0.01$, Fig. 4A) and immunofluorescence staining
( $p<0.01$, Fig. 4C). Besides, the results of Western blot and the activity assay showed that the ROCK2 protein expression ( $p<0.05$, Fig. 4A) and activity ( $p<0.01$, Fig. 4B) of APP/PS1 mice were also markedly increased. As expected, the elevated expression of ROCK2 ( $p<0.01$, Fig. 4A), p-ROCK2 $(p<0.01$, and $p<0.05$, respectively) and ROCK 2 activity $(p<0.001$, Fig. 4B) were largely reversed by Fasudil treatment. When compared with that of WT mice, the results showed ROCK2 expression was slightly declined in the Fasudil treatment group, but there was no sig- 

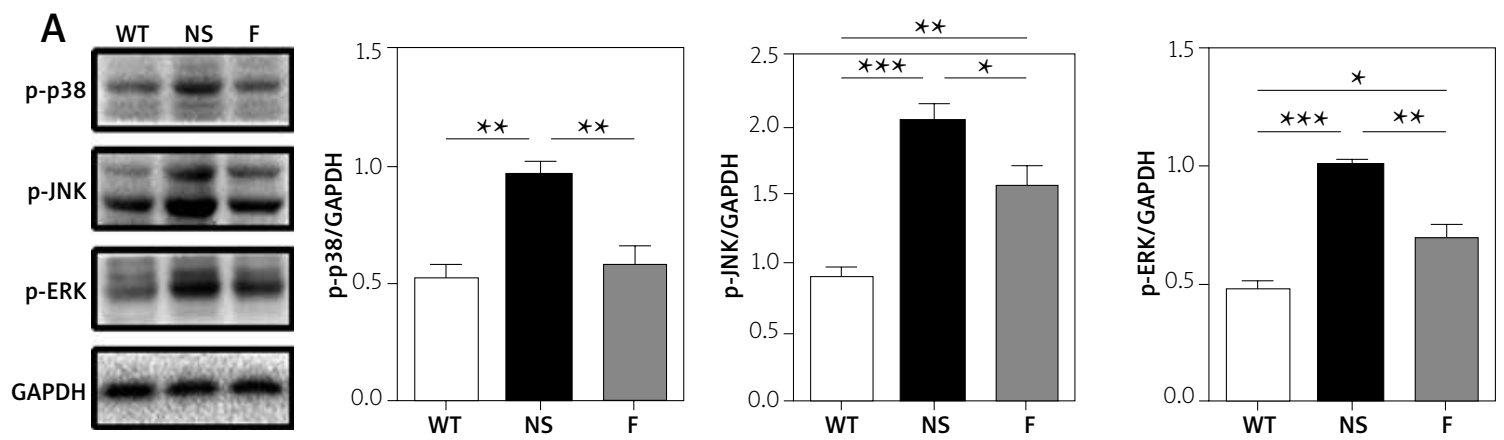

B
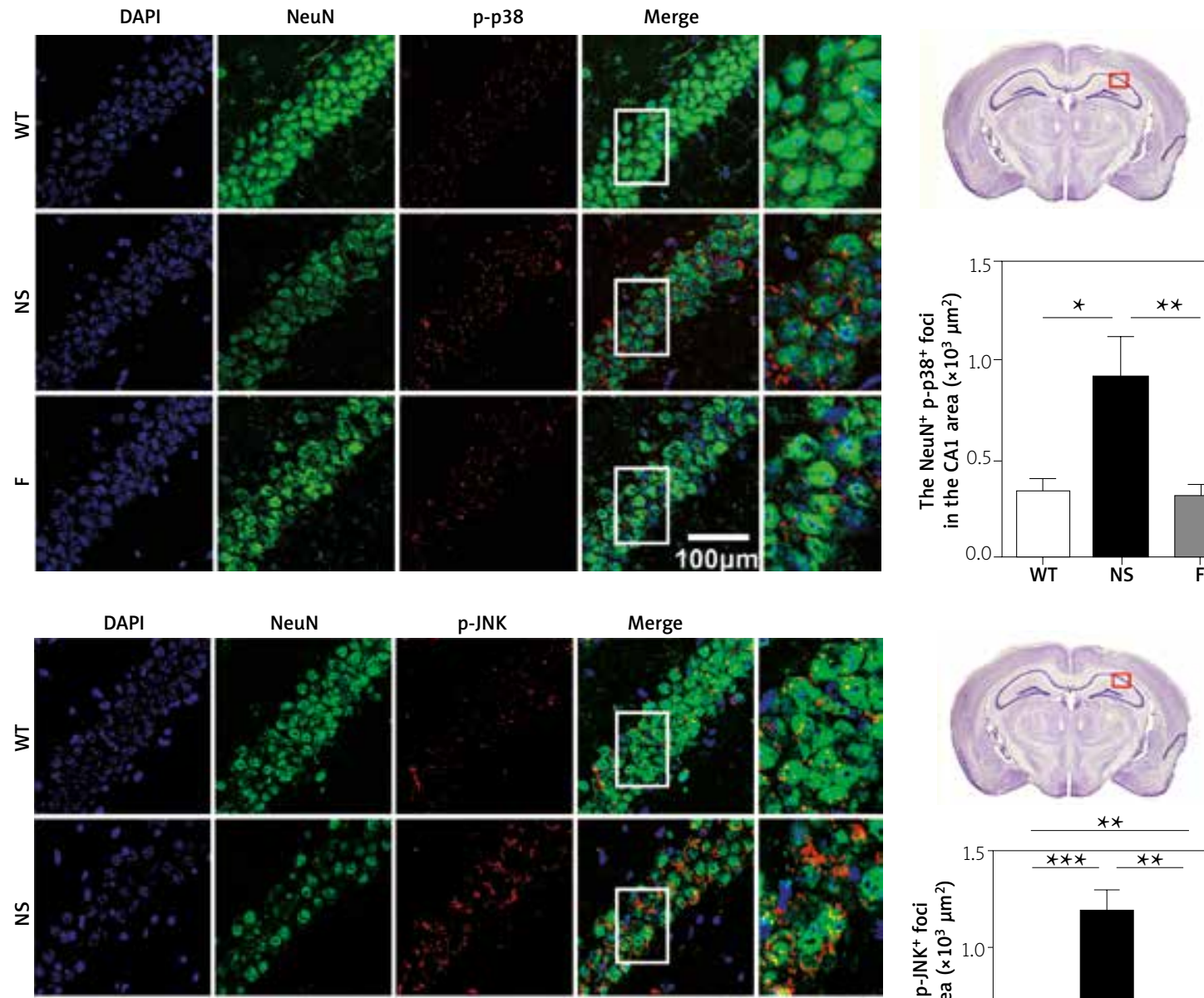

Merge
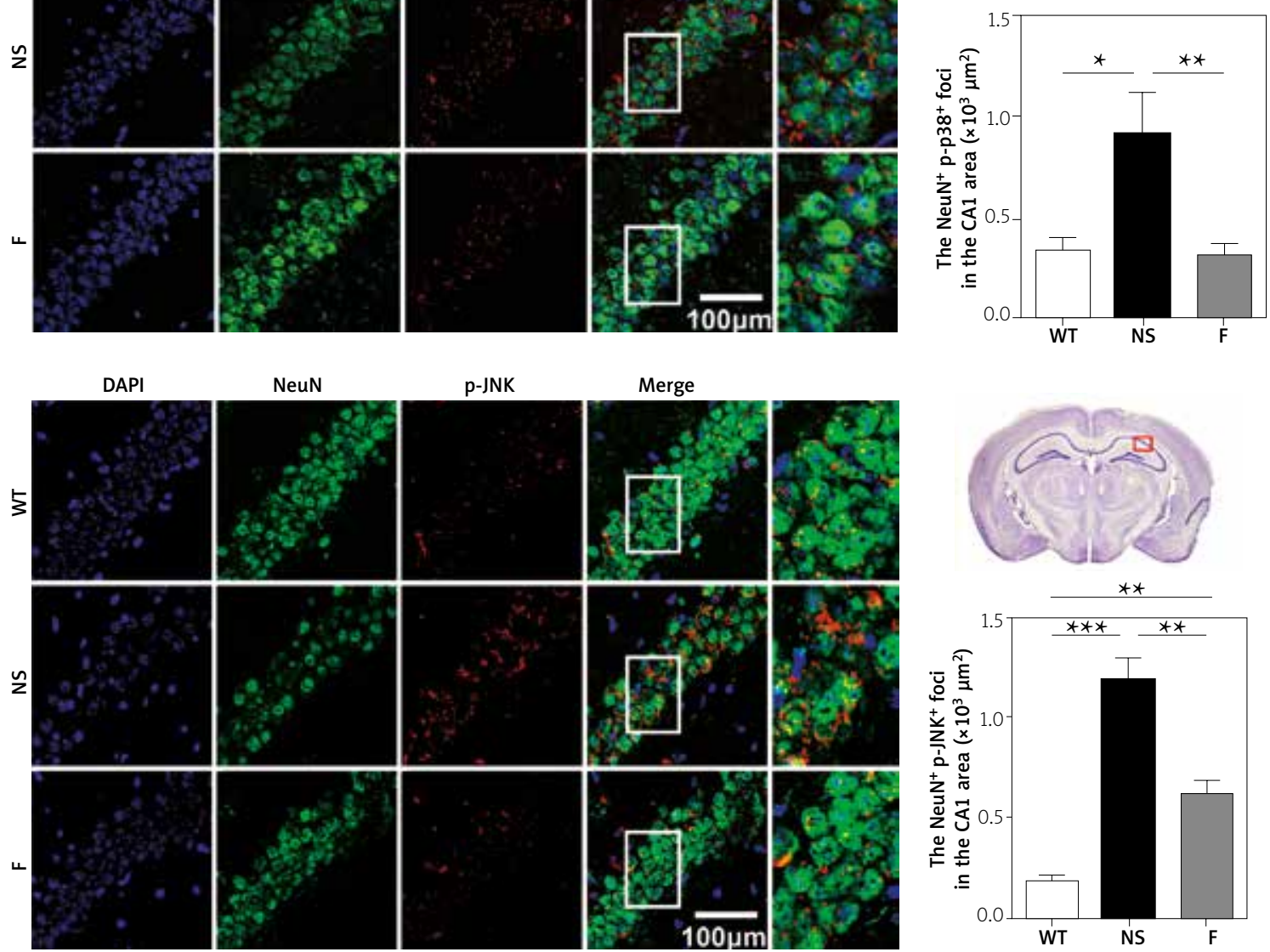

Fig. 5. Fasudil attenuated the phosphorylation level of MAPKs. A) The Western blot results and corresponding quantitative analysis for p-p38 (Y182), p-JNK (T183+T183+T221), and p-ERK (Tyr204) in hippocampus tissue homogenate. GAPDH was used as an internal loading control. B) The expression area of p-p38 (Y182), p-JNK (T183+T183+T221), and p-ERK (Tyr204) (red) positive neurons (green) in CA1 or DG zone of the hippocampus by double immunofluorescence and quantitative analysis (40x, Scale bar: $100 \mu \mathrm{m})$. Data were expressed as mean \pm SD, $n=4$ per group. Significant differences between groups were presented as ${ }^{*} p<0.05,{ }^{* *} p<0.01,{ }^{* *} p<0.001$, one-way ANOVA followed by Tukey's post-hoc test. 

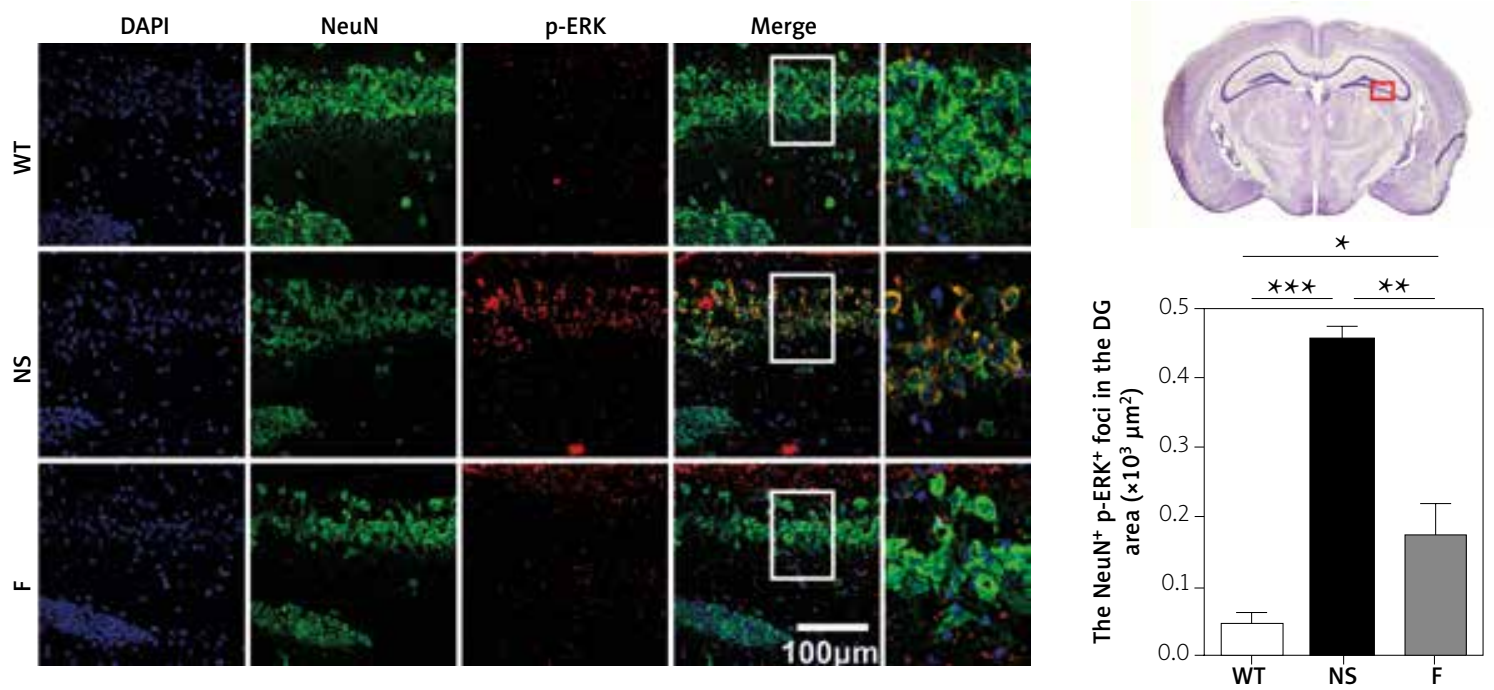

Fig. 5. Cont. B) The expression area of p-p38 (Y182), p-JNK (T183+T183+T221), and p-ERK (Tyr204) (red) positive neurons (green) in CA1 or DG zone of the hippocampus by double immunofluorescence and quantitative analysis $(40 \times$, Scale bar: $100 \mu \mathrm{m})$. Data were expressed as mean \pm SD, $n=4$ per group. Significant differences between groups were presented as ${ }^{*} p<0.05$, ${ }^{* *} p<0.01$, ${ }^{* * *} p<0.001$, one-way ANOVA followed by Tukey's post-hoc test.

nificant difference $(p>0.05)$. Our results confirmed that Fasudil can remarkably restrain the activity of Rho kinase and might be a potentially pharmacological target related to neurodegenerative disorders.

\section{Fasudil inhibited MAPK pathway in APP/PS1 mice}

There has already been a lot of studies focusing on the MAPK pathway and the importance of MAPK in $A D$ has become widely accepted in recent years [29]. In Figure 5, we showed Western blot analysis and immunofluorescence staining of p-p38 (Y182), p-JNK (T183+T183+T221), and p-ERK (Tyr204) among three groups. The significant increases of p-p38 ( $p<0.01$, and $p<0.05$, respectively) and p-JNK ( $p<0.001$, and $p<0.001$, respectively) were observed in the hippocampal CA1 area of APP/PS1 mice as compared with those in WT mice. Similarly, the phosphorylation level of ERK $(p<0.001$, and $p<0.001$, respectively) was also much higher than that of the WT group, especially in the hippocampal DG area. However, Fasudil effectively inhibited the phosphorylation of p38 $(p<0.01$, and $p<0.01$, respectively), JNK ( $p<0.05$, and $p<0.01$, respectively) and $\operatorname{ERK}(p<0.01$, and $p<0.01$, respectively). There were no significant changes in $p$-p38 expres- sion ( $p>0.05$, Fig. 5A) and immunofluorescence ( $p>0.05$, Fig. 5B) in the Fasudil treated group when compared with the wild type mice, although the expression of $\mathrm{p}$-JNK $(p<0.01$, and $p<0.01$, respectively), and $p$-ERK ( $p<0.05$, and $p<0.05$, respectively) were decreased which did not reach the normal levels. These data indicated that the mechanism of Fasudil could have inhibited the MAPK signalling pathway.

\section{Fasudil activated Nrf2 pathway in APP/PS1 mice}

As a key transcription factor, Nrf2 regulates its downstream molecules expression related to the anti-oxidative stress response, hence controls the cellular defence mechanism [20]. Among the protein extracts of hippocampus in APP/PS1 Tg mice, the expression of Nrf2 $(p<0.01$, Fig. $6 \mathrm{~A})$ and its downstream products including HO-1, NQO1, and SOD2 $(p<0.01$, $p<0.01$, and $p<0.01$, respectively, Fig. $6 \mathrm{~A})$ were obviously decreased compared with WT mice. Similar results were observed by using immunofluorescence staining analyses $(p<0.05, p<0.05, p<0.01$, and $p<0.01$, respectively, Fig. 6B). After Fasudil treatment, the results of Western blot and immunofluorescence staining showed that Nrf2 was translocated 

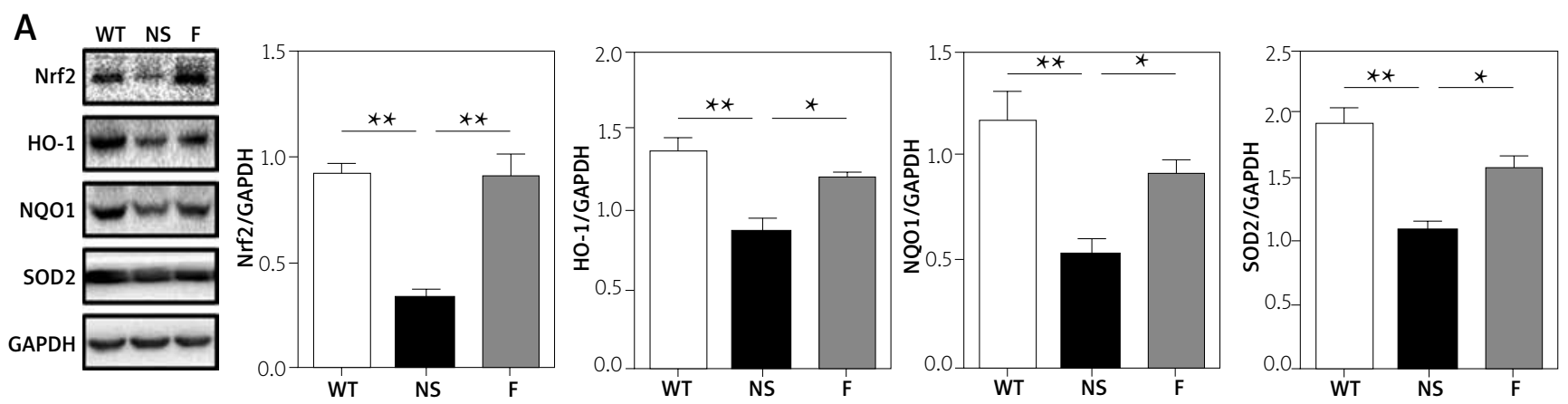

B
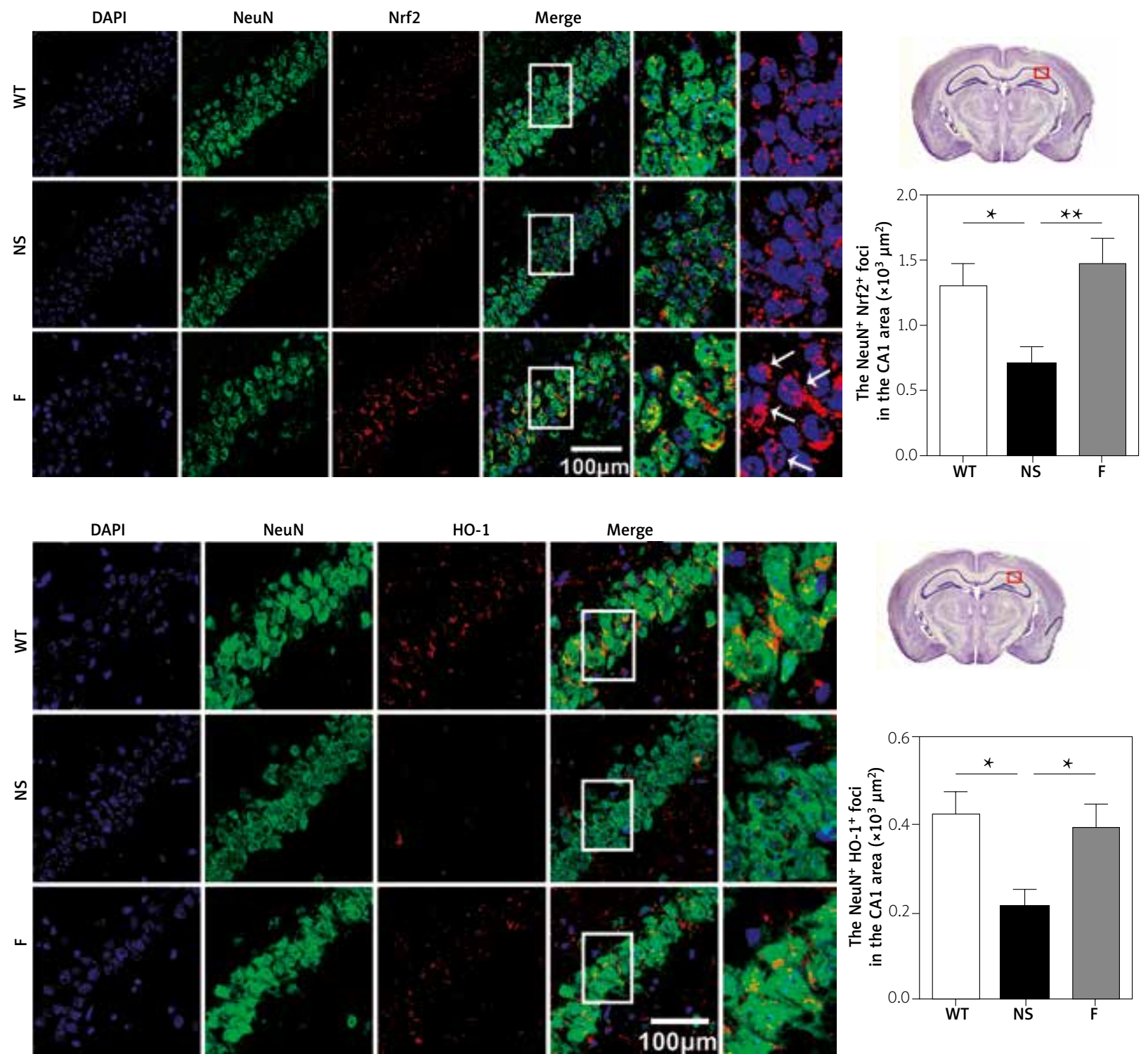

Fig. 6. Fasudil improved the expression of Nrf2 and its downstream antioxidant molecules. A) The expressions of Nrf2, HO-1, NQO1, and SOD2 in the hippocampus among three groups were detected by Western blot. The levels of quantitative proteins were normalized to the levels of GAPDH. B) The hippocampal CA1 neurons were subjected to double immunofluorescence staining by anti-Nrf2, anti-HO-1, anti-NQO1 anti-SOD2 (red), and anti-NeuN (green) $(40 \times$, Scale bar: $100 \mu \mathrm{m})$. Quantitative data were expressed as mean \pm SD, $n=4$ per group. Significant differences between groups were presented as ${ }^{*} p<0.05,{ }^{* *} p<0.01$, ${ }^{* *} p<0.001$, one-way ANOVA followed by Tukey's post-hoc test. 

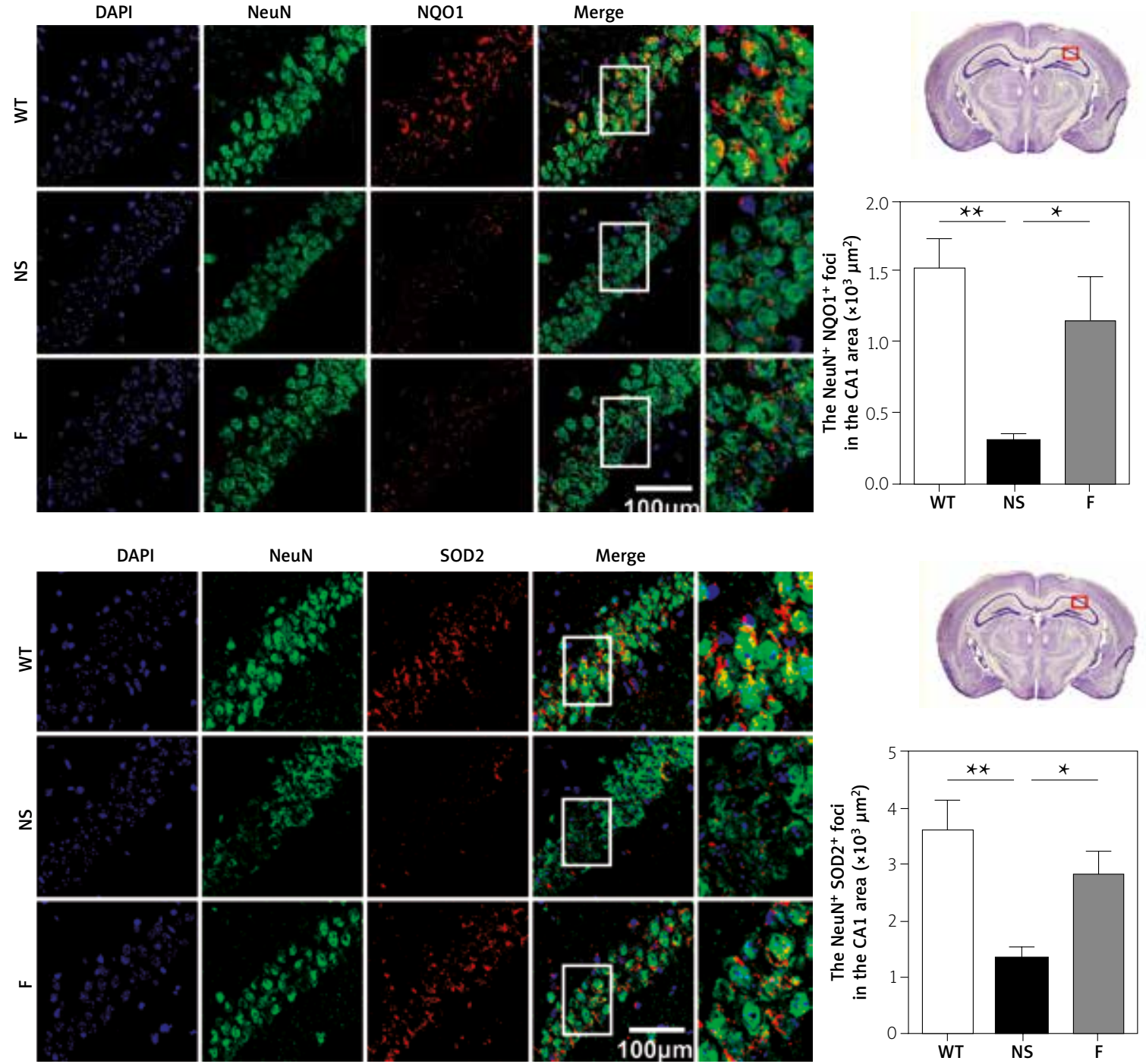

Fig. 6. Cont. B) The hippocampal CA1 neurons were subjected to double immunofluorescence staining by antiNrf2, anti-HO-1, anti-NQO1 anti-SOD2 (red), and anti-NeuN (green) (40x, Scale bar: $100 \mu \mathrm{m})$. Quantitative data were expressed as mean $\pm \mathrm{SD}, n=4$ per group. Significant differences between groups were presented as ${ }^{*} p<$ $0.05,{ }^{* *} p<0.01,{ }^{* *} p<0.001$, one-way ANOVA followed by Tukey's post-hoc test.

into neuron nucleus leading to the nuclear accumulation of $\mathrm{Nrf2}(p<0.01$, and $p<0.01$, respectively) to facilitate the production of numerous antioxidant enzymes such as HO-1 ( $p<0.05$, and $p<0.05$, respectively), NQO1 ( $p<0.05$, and $p<0.05$, respectively), and SOD2 ( $p<0.05$, and $p<0.05$, respectively). However, these increases did not reach the normal levels as shown in Figure $6 A$ and $B$, in which there were no significant changes observed (all $p>0.05$ ) in Nrf2, HO-1, NQO1, and SOD2 in Fasudil treated APP/PS1 mice compared with the WT group. These results suggest that Fasudil could have strongly activated the Nrf2 signalling pathway in APP/PS1 mice.

\section{Discussion}

Alzheimer's disease is well known as one of the common clinical diseases which has a rapidly increasing rate of incidence and death year by year, but its pathogenesis has not been completely elucidated so far and there is no effective drug for prevention and treatment. Hence, to find effective and safe agents has become necessary in recent years. 

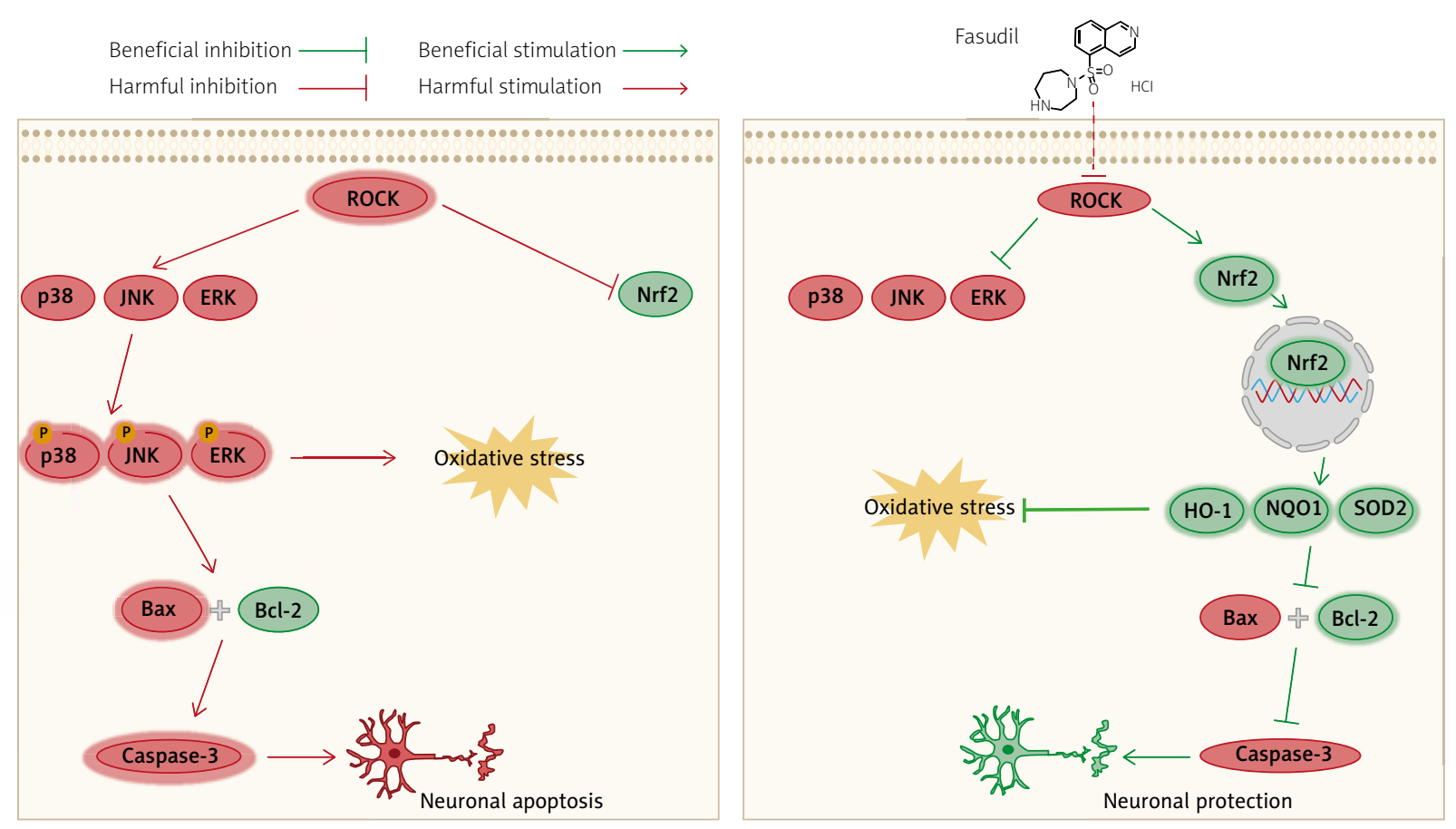

Fig. 7. Schematic diagram of the hypothesized signalling pathways summarizing the therapeutic effects of Fasudil against AD. Fasudil exhibited strong beneficial effects which may be mainly due to effectively suppressing the activity of ROCK and subsequently regulating MAPK signalling as well as accelerating the accumulation of nuclear Nrf2, eventually leading to relief of oxidative damage and neuronal apoptosis.

Oxidative stress emerges at the earliest stage of $A D$ before symptoms appear and plays an important role. It is generally accepted that, in $A D$, the antioxidant system breaks down and lipid peroxidation accumulates. Mitochondrion is both the major source of oxidative products and the main impaired organelle attacked by these products [43]. The imbalance between proapoptotic proteins (Bax) and antiapoptotic protein ( $\mathrm{Bcl}-2)$ is also responsible for the abnormal mitochondria permeability and the activation of the final executor of apoptosis, caspase-3, finally leading to the widespread apoptosis of neurons in the hippocampus. Neuronal apoptosis is an essential factor for neuronal death, which is closely related to the progression of $A D$. In the present study, Fasudil inhibited oxidative stress and alleviated neuronal apoptosis. This was in line with our previous reports about the effect of Fasudil on the MPTPmouse model of Parkinson's disease [41].

ROCK is an important target which is closely relevant to several physiological and pathological processes of many diseases. Nowadays, it is widely acknowledged that abnormal activation of ROCK has been regarded as an important factor which accelerates the occurrence and development of AD. ROCK activation increases $A \beta$ production [42], decreases neurite outgrowth of neurons and causes other pathological changes [36]. As we reported previously, Fasudil inhibited the expression of ROCK2 and reduced typical pathological changes of $A D$, such as $A \beta$ deposition and Tau protein phosphorylation, regulated the peripheral immune system, inhibited the inflammatory responses and increased the neurotrophic factors in vivo [37-39]. In another in vitro study, we found that Fasudil treatment attenuated $A \beta_{1-42}$-induced apoptosis by regulating the MAPK pathway in primary cultures of hippocampal neurons [13]. In addition, there is evidence that the activation of ROCK not only elevates the level of oxidative stress $[19,23]$, but also can be caused by the status of oxidative stress, reinforcing a vicious cycle and ultimately causing neuronal death [34]. Our results reported in both this study and the previous reports [41] suggested that ROCK inhibitors have potent anti-oxidant and anti-apoptotic effects.

Recent investigations showed that the increased ROCK activity was required for neurodegeneration and then triggered several downstream pathways. 
Oxidative stress emerges at the earliest stage of $A D$ before symptoms appear and plays an important role. It is generally accepted that, in $A D$, the antioxidant system breaks down and lipid peroxidation accumulates. Mitochondrion is both the major source of oxidative products and the main impaired organelle attacked by these products [43]. The imbalance between proapoptotic proteins (Bax) and antiapoptotic protein ( $\mathrm{Bcl}-2)$ is also responsible for the abnormal mitochondria permeability and the activation of the final executor of apoptosis, caspase-3, finally leading to the widespread apoptosis of neurons in the hippocampus. Neuronal apoptosis is an essential factor for neuronal death, which is closely related to the progression of AD. In the present study, Fasudil inhibited oxidative stress and alleviated neuronal apoptosis. This was in line with our previous reports about the effect of Fasudil on the MPTPmouse model of Parkinson's disease [41].

ROCK is an important target which is closely relevant to several physiological and pathological processes of many diseases. Nowadays, it is widely acknowledged that abnormal activation of ROCK has been regarded as an important factor which accelerates the occurrence and development of AD. ROCK activation increases $A \beta$ production [42], decreases neurite outgrowth of neurons and causes other pathological changes [36]. As we reported previously, Fasudil inhibited the expression of ROCK 2 and reduced typical pathological changes of $A D$, such as $A B$ deposition and Tau protein phosphorylation, regulated the peripheral immune system, inhibited the inflammatory responses and increased the neurotrophic factors in vivo [37-39]. In another in vitro study, we found that Fasudil treatment attenuated $A \beta_{1-42}$-induced apoptosis by regulating the MAPK pathway in primary cultures of hippocampal neurons [13]. In addition, there is evidence that the activation of ROCK not only elevates the level of oxidative stress $[19,23]$, but also can be caused by the status of oxidative stress, reinforcing a vicious cycle and ultimately causing neuronal death [34]. Our results reported in both this study and the previous reports [41] suggested that ROCK inhibitors have potent anti-oxidant and anti-apoptotic effects.

Recent investigations showed that the increased ROCK activity was required for neurodegeneration and then triggered several downstream pathways. The MAPKs, p38, JNK and ERK, were identified as the downstream substrates of ROCK [32]. Several lines of evidence showed that some external stimuli including the elevated ROCK activity could activate these MAPK molecules by facilitating their phosphorylation, finally rise up to oxidative stress $[12,14]$ and cell apoptosis [33]. In the present vivo trial, we further demonstrated that Fasudil effectively inhibited the activation of $\mathrm{p3}$, JNK, and ERK in APP/PS1 transgenic mice. Therefore, we believed that Fasudil may protect neurons by decreasing the activation of the MAPK signalling pathway in vivo.

Oxidative stress is very strongly related to the occurrence of aging and age-related disorders. Nrf2 is a critical transcription factor which is correlated with the expression of several cyto-protective target genes such as HO-1, NQO1 and SOD2, allowing to relieve oxidative stress and maintain cellular homeostasis. It was previously reported that $A \beta$-induced oxidative damage in $\mathrm{SH}-\mathrm{SY} 5 \mathrm{Y}$ cells could be reduced by activating the Nrf2 signalling pathway [40]. In accordance with our previous reports, Fasudil was found to lead to the upregulation of Nrf2 and induction of antioxidant molecules in primary cultures of hippocampal neurons [13], so it was believed that Fasudil may exert neuroprotective effects through activating the Nrf2 signalling pathway which promotes the anti-apoptosis ability of the brain. Here, as revealed by our present study, Fasudil also upregulated the expression of $\mathrm{Nrf2}$ and facilitated Nrf2 being translocated into the nuclei in APP/PS1 mice. Furthermore, the increase in ROCK activity leads to the overproduction of free radicals and results in the oxidative stress $[5,19,23]$ that will trigger the activation of Nrf2 by feedback response $[3,16]$. And the most important is that the elevated level of Nrf2 could induce the partial reduction of the aberrant activation of ROCK [34].

In the study, APP/PS1 double transgenic mice were used as a dementia model in order to examine the antioxidative effects of Fasudil and investigate related cytological and molecular mechanisms. Based on the results of Western blot and immunofluorescence, we found that Fasudil treatment improved the cognitive function, inhibited oxidative stress and reduced hippocampus neuronal apoptosis in APP/PS1 mice which may be mediated by the ROCK, MAPK and Nrf2 signalling pathways. Moreover, the phosphorylation levels of p38, JNK, and ERK were dramatically suppressed by Fasudil treatment. Meanwhile, the expression of anti-apoptotic protein $\mathrm{Bcl}-2$ was enhanced while the expression 
of pro-apoptotic protein Bax and cleaved caspase-3 were decreased. Thus, our results provided further evidence that the selective inhibitor of ROCK, Fasudil, might be a promising drug candidate to deal with AD-related damage by inhibiting ROCK/MAPK and activating Nrf2, as shown in Figure 7.

Although the data shown here are some of the first to report that Fasudil protects against AD possibly via the inhibition of ROCK/MAPK and the activation of Nrf2 signalling pathway, the evidence is still very preliminary and limited. Further research and pre-clinical studies are needed to determine the role of Fasudil in the clinical management of AD.

\section{Conclusions}

In conclusion, our present data have shown that Fasudil might improve cognitive function, restrain oxidative stress, and suppress neuronal apoptosis via inhibiting ROCK/MAPK and activating the Nrf2 pathway in AD mice. Fasudil appears to have tremendous potential to prevent or treat $A D$ in the future.

\section{Acknowledgements}

This work was supported by research grants from the National Natural Science Foundation of China (81473577, 81471412 and 82004028), and Scientific and Technological Innovation Team of Integrated Chinese and Western Medicine for the Prevention and Treatment on Nervous System Diseases, Shanxi University of Chinese Medicine (2018TD-012), Natural Fund Project of Shanxi Province (201901D111334 and 201901D211538), Research Project Supported by Shanxi Scholarship Council of China (2014-7), Project of Shanxi Province Platform Base (201805D131005 and 201805D111009), Shanxi Province Key R \& D Plan (2106ZD0505) and Platform Base Plan Project of Datong (2019198).

\section{Availability of data and materials}

The datasets used and/or analysed during the current study are available from the corresponding author on reasonable request.

\section{Ethics approval and consent to participate}

All experimental procedures of animals were approved by the Ethics Committee of Shanxi Datong University, Datong, China.

\section{Disclosure}

The authors report no conflict of interest.

\section{References}

1. Arthur JS, Ley SC. Mitogen-activated protein kinases in innate immunity. Nat Rev Immunol 2013; 13: 679-692.

2. Bahn G, Jo DG. Therapeutic approaches to Alzheimer's disease through modulation of NRF2. Neuromolecular Med 2019; 21: 1-11.

3. Bellezza I, Giambanco I, Minelli A, Donato R. Nrf2-Keap1 signaling in oxidative and reductive stress. Biochim Biophys Acta Mol Cell Res 2018; 1865: 721-733.

4. Burrows EL, McOmish CE, Buret LS, Van den Buuse M, Hannan AJ. Environmental enrichment ameliorates behavioral impairments modeling schizophrenia in mice lacking metabotropic glutamate receptor 5. Neuropsychopharmacol 2015; 40: 1947-1956.

5. Calò LA, Vertolli U, Pagnin E, Ravarotto V, Davis PA, Lupia M, Naso E, Maiolino G, Naso A. Increased rho kinase activity in mononuclear cells of dialysis and stage 3-4 chronic kidney disease patients with left ventricular hypertrophy: Cardiovascular risk implications. Life Sci 2016; 148: 80-85.

6. Chen M, Liu A, Ouyang Y, Huang Y, Chao X, Pi R. Fasudil and its analogs: a new powerful weapon in the long war against central nervous system disorders? Expert Opin Investig Drugs 2013; 22: 537-550

7. Chen YG. Research progress in the pathogenesis of Alzheimer's disease. Chin Med J 2018; 131: 1618-1624.

8. Chong CM, Ai N, Lee SM. ROCK in CNS: different roles of isoforms and therapeutic target for neurodegenerative disorders. Curr Drug Targets 2017; 18: 455-462.

9. Cobley JN, Fiorello ML, Bailey DM. 13 reasons why the brain is susceptible to oxidative stress. Redox Biol 2018; 15: 490-503.

10. Ding J, Li QY, Yu JZ, Wang X, Sun CH, Lu CZ, Xiao BG, Wang X, Sun CH, Lu CZ, Xiao BG. Fasudil, a Rho kinase inhibitor, drives mobilization of adult neural stem cells after hypoxia/reoxygenation injury in mice. Mol Cell Neurosci 2010; 43: 201-208.

11. Dinkova-Kostova AT, Kostov RV, Kazantsev AG. The role of Nrf2 signaling in counteracting neurodegenerative diseases. FEBS I 2018; 285: 3576-3590

12. Gan P, Gao Z, Zhao X, Qi G. Surfactin inducing mitochondria-dependent ROS to activate MAPKs, NF-KB and inflammasomes in macrophages for adjuvant activity. Sci Rep 2016; 6: 39303.

13. Gao Y, Yan Y, Fang Q, Zhang N, Kumar G, Zhang J, Song LJ, Yu J, Zhao L, Zhang HT, Ma CG. The Rho kinase inhibitor fasudil attenuates $A \beta(1-42)$-induced apoptosis via the ASK1/JNK signal pathway in primary cultures of hippocampal neurons. Metab Brain Dis 2019; 34: 1787-1801.

14. Gong L, Lei Y, Liu Y, Tan F, Li S, Wang X, Xu M, Cai W, Du B, Xu F, Zhou Y, Han H, Sun H, Qiu L. Vaccarin prevents ox-LDL-induced HUVEC EndMT, inflammation and apoptosis by suppressing ROS/p38 MAPK signaling. Am I Transl Res 2019; 11: 2140-2154.

15. Gu QF, Yu JZ, Wu H, Li YH, Liu CY, Feng L, Zhang GX, Xiao BG, Ma CG. Therapeutic effect of Rho kinase inhibitor FSD-C10 in a mouse model of Alzheimer's disease. Exp Ther Med 2018; 16: 3929-3938. 
16. Guan P, Liang Y, Wang N. Fasudil alleviates pressure overload-induced heart failure by activating Nrf2-mediated antioxidant responses. J Cell Biochem Suppl 2018; 119: 6452-6460.

17. Islam MT. Oxidative stress and mitochondrial dysfunction-linked neurodegenerative disorders. Neurol Res 2017; 39 73-82.

18. Jazvinšćak Jembrek M, Hof PR, Šimić G. Ceramides in Alzheimer's disease: Key mediators of neuronal apoptosis induced by oxidative stress and $A \beta$ accumulation. Oxid Med Cell Longev 2015; 2015: 346783.

19. Kang HT, Park JT, Choi K, Choi HJC, Jung CW, Kim GR, Lee YS, Park SC. Chemical screening identifies ROCK as a target for recovering mitochondrial function in Hutchinson-Gilford progeria syndrome. Aging Cell 2017; 16: 541-550.

20. Kasai S, Shimizu S, Tatara Y, Mimura J, Itoh K. Regulation of Nrf2 by mitochondrial reactive oxygen species in physiology and pathology. Biomolecules 2020; 10: 320.

21. Kim EK, Choi EJ. Compromised MAPK signaling in human diseases: an update. Arch Toxicol Suppl 2015; 89: 867-882.

22. Koch JC, Tatenhorst L, Roser AE, Saal KA, Tönges L, Lingor P. ROCK inhibition in models of neurodegeneration and its potential for clinical translation. Pulm Pharmacol Ther 2018; 189: 1-21.

23. Lu W, Kang J, Hu K, Tang S, Zhou X, Xu L, Li Y, Yu S. The role of the Nox4-derived ROS-mediated RhoA/Rho kinase pathway in rat hypertension induced by chronic intermittent hypoxia. Sleep Breath 2017; 21: 667-677.

24. Narumiya S, Thumkeo D. Rho signaling research: history, current status and future directions. FEBS Lett 2018; 592: 1763 1776.

25. Padurariu M, Ciobica A, Mavroudis I, Fotiou D, Baloyannis S. Hippocampal neuronal loss in the CA1 and CA3 areas of Alzheimer's disease patients. Psychiatr Danub 2012; 24: 152-158.

26. Prince $M$, Comas-Herrera A, Knapp M, Guerchet M, Karagiannidou M. World Alzheimer report 2016: improving healthcare for people living with dementia: coverage, quality and costs now and in the future. Alzheimer's Disease International (ADI), London 2016.

27. Radi E, Formichi P, Battisti C, Federico A. Apoptosis and oxidative stress in neurodegenerative diseases. Am J Alzheimers Dis 2014; 42: S125-152.

28. Shahbazi R, Baradaran B, Khordadmehr M, Safaei S, Baghbanzadeh A, Jigari F, Ezzati H. Targeting ROCK signaling in health, malignant and non-malignant diseases. Immunol Lett 2020; 219: 15-26.

29. Song J, Park KA, Lee WT, Lee JE. Apoptosis signal regulating kinase 1 (ASK1): potential as a therapeutic target for Alzheimer's disease. Int J Mol Sci 2014; 15: 2119-2129.

30. Song Y, Chen X, Wang LY, Gao W, Zhu MJ. Rho kinase inhibitor fasudil protects against -amyloid-induced hippocampal neurodegeneration in rats. CNS Neurosci Ther 2013; 19: 603-610.

31. Tadokoro K, Ohta Y, Inufusa H, Loon AFN, Abe K. Prevention of cognitive decline in Alzheimer's disease by novel antioxidative supplements. Int J Mol Sci 2020; 21: 1974.

32. Wang C, Choi YH, Xian Z, Zheng M, Piao H, Yan G. Aloperine suppresses allergic airway inflammation through NF- B, MAPK, and Nrf2/HO-1 signaling pathways in mice. Int Immunopharmacol 2018; 65: 571-579.
33. Wang X, Mao R, Chen W. FSD-C10 shows therapeutic effects in suppressing oxidized low-density lipoprotein (ox-LDL)-induced human brain microvascular endothelial cells apoptosis via Rho-associated coiled-coil kinase (ROCK)/mitogen-activated protein kinase (MAPK) signaling. Med Sci Monit 2018; 24: 5509-5516.

34. Wang X, Zhao X, Feng T, Jin G, Li Z. Rutin prevents high glucose-induced renal glomerular endothelial hyperpermeability by inhibiting the ROS/Rhoa/ROCK signaling pathway. Planta Med 2016; 82: 1252-1257.

35. Weller J, Budson A. Current understanding of Alzheimer's disease diagnosis and treatment. F1000Res 2018; 7: 1161.

36. Xin YL, Yu JZ, Yang XW, Liu CY, Li YH, Feng L, Chai Z, Yang WF, Wang Q, Jiang WJ, Zhang GX, Xiao BG, Ma CG. FSD-C10: A more promising novel ROCK inhibitor than Fasudil for treatment of CNS autoimmunity. Biosci Rep 2015; 35: e00247.

37. Yan Y, Yu J, Gao Y, Kumar G, Guo M, Zhao Y, Fang Q, Zhang H, Yu J, Jiang Y, Zhang HT, Ma CG. Therapeutic potentials of the Rho kinase inhibitor Fasudil in experimental autoimmune encephalomyelitis and the related mechanisms. Metab Brain Dis 2019; 34: 377-384.

38. Yu JZ, Li YH, Liu CY, Wang Q, Gu QF, Wang HQ, Zhang GX, Xiao BG, Ma CG. Multitarget therapeutic effect of Fasudil in APP/PS1 transgenic mice. CNS Neurosci Ther 2017; 16: 199-209.

39. Yu JZ, Yan YQ, Gu QF, Kumar G, Yu HQ, Zhao Y, Liu C, Gao Y, Chai Z, Chumber J, Xiao BG, Zhang GX, Zhang HT, Jiang Y, Ma CG. Fasudil in combination with bone marrow stromal cells (BMSCs) attenuates Alzheimer's disease-related changes through the regulation of the peripheral immune system. Front Aging Neurosci 2018; 10: 216.

40. Zhang L, Guo Y, Wang H, Zhao L, Ma Z, Li T, Liu J, Sun M, Jian Y, Yao L, Du Y, Zhang G. Edaravone reduces $A \beta$-induced oxidative damage in SH-SY5Y cells by activating the Nrf2/ARE signaling pathway. Life Sci 2019; 221: 259-266.

41. Zhao YF, Zhang Q, Xi JY, Li YH, Ma CG, Xiao BG. Multitarget intervention of Fasudil in the neuroprotection of dopaminergic neurons in MPTP-mouse model of Parkinson's disease. J Neurol Sci Turk 2015; 353: 28-37.

42. Zhou Y, Su Y, Li B, Liu F, Ryder JW, Wu X, Gonzalez-DeWhitt PA, Gelfanova V, Hale JE, May PC, Paul SM, Ni B. Nonsteroidal anti-inflammatory drugs can lower amyloidogenic Abeta 42 by inhibiting Rho. Science 2003; 302: 1215-1217.

43. Zhou Y, Xie N, Li L, Zou Y, Zhang X, Dong M. Puerarin alleviates cognitive impairment and oxidative stress in APP/PS1 transgenic mice. Int J Neuropsychopharmacol 2014; 17: 635-644. 